\title{
About Fixed Points in CAT(0) Spaces under a Combined Structure of Two Self-Mappings
}

\author{
Manuel De la Sen \\ Institute of Research and Development of Processes, University of the Basque Country, Campus of Leioa 48940, Bizkaia, Spain \\ Correspondence should be addressed to Manuel De la Sen; manuel.delasen@ehu.eus
}

Received 24 January 2017; Revised 28 April 2017; Accepted 8 June 2017; Published 19 July 2017

Academic Editor: Willi Freeden

Copyright ( 2017 Manuel De la Sen. This is an open access article distributed under the Creative Commons Attribution License, which permits unrestricted use, distribution, and reproduction in any medium, provided the original work is properly cited.

\begin{abstract}
This paper investigates some fixed point-related questions including the sequence boundedness and convergence properties of mappings $Q_{t}(S x, T y)=t S x \oplus(1-t) T y$ defined in CAT $(0)$ spaces, which are parameterized by a scalar $t \in[0,1]$, where $S, T: X \rightarrow X$ are nonexpansive Lipschitz-continuous mappings and $(X, d)$ is a metric space which is a CAT $(0)$ space.
\end{abstract}

\section{Introduction}

A CAT $(k)$ space, where $k$ is a real number related to the curvature, is a type of metric space where triangles of potential vertices being each set of three points are thinner, that is, of length being less than or equal to the corresponding so-called comparison triangles (namely, those whose sides have the same lengths as the sides of the original triangle) in the model spaces. The curvature in a $\operatorname{CAT}(k)$ space is bounded from above by $k$. A particular case of CAT $(k)$ spaces $[1-3]$ is that arising when the curvature is bounded from above by 0 (CAT(0) spaces. See, for instance, [4-10]). Complete CAT(0) spaces, being often referred to as Hadamard spaces (in honor to Jacques Hadamard), generalize Hilbert spaces to the nonlinear framework. In Hadamard spaces, there is a unique geodesic path joining each pair of given points. In particular, a normed space is an Hadamard space if and only if it is a Hilbert space. It is well-known that Hadamard spaces satisfy the following inequality:

$$
d^{2}(z, m)+\frac{d^{2}(x, y)}{4} \leq \frac{1}{2}\left(d^{2}(z, x)+d^{2}(z, y)\right)
$$

for each of the given points $x, y, z$ and some point $m$, where $m$ is the unique midpoint of $x$ and $y$, that is, $d(x, y)=$ $2 d(x, m)=2 d(y, m)$, since Hadamard spaces are uniquely geodesic.

The study of geodesic paths is very relevant in spherical geometry, for instance, in the composition of trajectories through the Earth surface or in common planetary studies of distances. A metric space $(X, d)$ is a geodesic metric space if any two points $x, y \in X$ can be joined by an arc length parameterized continuous curve (geodesic segment) $\gamma$ : $[a, b] \rightarrow X, \gamma(a)=x, \gamma(b)=y$ whose length $L(\gamma)=$ $d(x, y)$. It is well-known that a geodesic metric space $(X, d)$ is a CAT(0) space if every geodesic triangle $\Delta$ in $X$ satisfies the CAT(0) inequality; namely, the distance between any two points of such a triangle is less than or equal to the distance between the corresponding points of the model triangle in the Euclidean space, that is, a triangle $\bar{\Delta}$ with sides of the same length as the sides of $\Delta$ and then of the same perimeter. Therefore, the study of the metric properties of CAT( 0 ) spaces has a major importance. In general, a geodesic metric space $(X, d)$ is a $\operatorname{CAT}(k)$ space if every geodesic triangle in $X$ with perimeter less than $2 D_{k}$ satisfies the $\operatorname{CAT}(k)$ inequality. The so-called CAT(0) spaces are of curvature $k=0$ and they are particular spaces of the most general CAT $(k)$ spaces of curvature $k \in \mathbf{R}$. To fix some general basic ideas, let us denote by $M_{k}$ the unique 2-dimensional Riemannian manifold with constant curvature $k$ of diameter $D_{k}$ being $+\infty$ if $k \leq 0$ and $\pi / \sqrt{k}$ for $k>0$. The $\mathrm{CAT}(k)$ spaces are those whose geodesic triangles $\Delta$, that is, those having geodesic segments as its sides, satisfy the so-called $\operatorname{CAT}(k)$ inequality; namely, the distances between points of $\Delta$ are less than or equal to the distances between the corresponding points in their comparison triangles $\Delta^{\prime}$ in the model space $M_{k}$ (i.e., those triangles whose sides have the same lengths as their counterparts as 
their corresponding $\Delta$-triangles). Note that if the metric space $(X, d)$ is a $\operatorname{CAT}(k)$ space, then there is a unique geodesic segment with joins $x$ and $y$ (with $d(x, y)<D_{k}$ if $k>0$ ) $[3,11]$.

Furthermore, it is well-known that CAT $(k)$ spaces are also $\operatorname{CAT}(\ell)$ spaces for $\ell>k$ and that $n$ - dimensional hyperbolic spaces with their usual metric are CAT $(-1)$ spaces and then CAT(0) spaces, whose simpler example is the $n$-dimensional Euclidean space with its usual metric, so also CAT(1) spaces as well, whose simpler example is the unit sphere. Other relevant CAT(0) spaces are the so-called Euclidean buildings, which are abstract simplicial complexes, and the so-called CAT(0) cube complexes, [12-15]. The first ones give a systematic procedure for geometric interpretation of semisimple Lie groups and the study of semisimple groups over general fields, while the second ones are important, for instance, in the modelling process of robot trajectories on eventually irregular surfaces with eventual obstacles and predesigned admissible corridors for trajectory tracking.

This paper has two subsequent body sections. Section 2 gives some relating results for some of the various existing concepts of convexity in metric spaces $(X, d)$ such as 1-convexity, $p$-convexity, midpoint convexity, convex structure, uniform convexity and near-uniform convexity, and Busemann curvature and its relation to convexity. Section 3 gives and proves some relevant properties related to uniform convexity and near-uniform convexity of geodesic metric CAT(0) spaces. It also studies mappings of the form $Q_{t}$ : $X \times X \rightarrow X$ parameterized by a scalar $t \in[0,1]$, defined by $Q_{t}(S x, T y)=t S x \oplus(1-t) T y$ in a metric space $(X, d)$, where $S, T: X \rightarrow X$ are Lipschitz-continuous while not necessarily contractive mappings; that is, the Lipschitz constants are not necessarily less than unity. In particular, the convergence properties of sequences built from such mappings are formally studied and some conditions of existence of fixed points are given. Some illustrative examples are also presented and discussed. The main aim and motivation of the formal study of the sequences generated by such parameterized mappings, which are constructed with two generator mappings on a metric space $(X, d)$, on $\mathrm{CAT}(0)$ spaces. The obtained and proved results rely on the boundedness and nonexpansive and contractive properties of the sequences generated by such mappings depending on the contractiveness/nonexpansiveness of both generator mappings. A potential application is the generation of admissible corridors whose extreme obstacle-free trajectories are defined by sequences generated by the two mentioned generator mappings. The generators of the corridor extreme trajectories are two primary mappings which define the studied parameterized mapping on the CAT(0) space. Those defined extreme trajectories can be bounded and/or convergent for each parameter value of the parameterized mapping of interest and could define the admissible trajectories of a robotic device or a movable body. One of the examples is concerned with this view on some potential applications.

\section{Notation}

$$
\begin{aligned}
& \mathbf{Z}_{0+}=\{z: z \geq 0\} . \\
& \mathbf{Z}_{+}=\{z: z>0\} . \\
& \mathbf{R}_{0+}=\{z: z \geq 0\} . \\
& \mathbf{R}_{+}=\{z: z>0\} .
\end{aligned}
$$

$c \ell A$ is the closure of the set $A$.

$\operatorname{con}\left\{x_{i}\right\}_{i \in I}$ is the closure of the convex hull of the family $\left\{x_{i}\right\}_{i \in I}$.

$\operatorname{Fix}(T)$ denotes the set of fixed points of a mapping $T: X \rightarrow X$.

\section{Some Preliminary Definitions and Results on Convexity, Uniform Convexity, and Curvature}

Let $(X, d)$ be a complete metric space. It is said that it admits (nonnecessarily unique) midpoints if for any $x, y \in X$, there is a $z=m(x, y) \in X$ such that $d(x, y)=2 d(x, z)=2 d(y, z)$. Such a point $z \in X$ is said to be a midpoint of $x$ and $y$ and $(X, d)$ is a geodesic space, $[16-18]$.

Definition 1 ( $p$-convexity $[16,19])$. Suppose a metric space $(X, d)$ which admits midpoints (or which has midpoints or which is midpoint convex). Then, $(X, d)$ is said to be $p$-convex for some $p \in[1, \infty]$ if, for each $x, y, z \in X$ and each midpoint $m(x, y) \in X$ of $x$ and $y$,

$$
d(m(x, y), z) \leq\left(\frac{1}{2}\right)^{1 / p}\left(d^{p}(x, z)+d^{p}(y, z)\right)^{1 / p}
$$

For the case $p=\infty$, the right-hand side of (2) is defined as a limit leading to $d(m(x, y), z) \leq \max (m(x, y), z)$. If $(X, d)$ is $\infty$-convex, it is equivalently said to be ball convex, while if it is 1-convex it is equivalently said to be distance convex [19]. $(X, d)$ is said to be strictly $p$-convex for $p \in(1, \infty]$, if the inequality is strict for $x \neq y$ and strictly 1-convex if the inequality is strict for $p=1$ if $d(x, y)>|d(x, z)-d(y, z)|$ [16].

\section{Definition 1 leads to the direct conclusion below.}

Assertion 2. If a metric space $(X, d)$ is midpoint convex, then it is 1-convex.

Assertion 3. If $(X, d)$ be $p$-convex for some $p \in[1, \infty)$ then, for any $x, y, z \in X$ and each midpoint $m(x, y) \in X$ of $x$ and $y$,

$$
\begin{aligned}
d(m(x, y), z) & \leq\left(\frac{1}{2}\right)^{1 / p}(d(x, z)+d(y, z)) \\
& \leq \frac{1}{2}(d(x, z)+d(y, z)) .
\end{aligned}
$$

Proof. It is direct from (2) and Minkowski inequality or by direct rearrangement of the power $1 / p$ to its inverse in the left-hand side of (2).

Inequality (2) leads to the following direct result. 
Assertion 4. If $(X, d)$ be $p$-convex for some $p \in[1, \infty)$ then, for any $x, y, z, w \in X$, each midpoint $m(x, y) \in X$ of $x$ and $y$ and each midpoint $m(z, w) \in X$ of $z$ and $w$,

$$
\begin{aligned}
& d(m(x, y), m(z, w)) \leq\left(\frac { 1 } { 4 } \left(d^{p}(x, z)+d^{p}(x, w)\right.\right. \\
& \left.\left.+d^{p}(y, z)+d^{p}(y, w)\right)\right)^{1 / p} .
\end{aligned}
$$

Proof. Note from (2) that

$$
d^{p}(y, m(x, z)) \leq \frac{1}{2}\left(d^{p}(y, x)+d^{p}(y, z)\right)
$$

and, one gets from (2) and (5) that

$$
\begin{gathered}
d(m(x, y), m(z, w)) \leq\left(\frac{1}{2}\right)^{1 / p}\left(d^{p}(x, m(z, w))\right. \\
\left.+d^{p}(y, m(z, w))\right)^{1 / p} \leq\left(\frac{1}{2}\right)^{1 / p}\left(\frac { 1 } { 2 } \left(d^{p}(x, z)\right.\right. \\
\left.\left.+d^{p}(x, w)+d^{p}(y, z)+d^{p}(y, w)\right)\right)^{1 / p}
\end{gathered}
$$

leading to the result.

Definition 5 ( $p$-Busemann curvature $[16,20])$. Suppose a metric space $(X, d)$ which admits midpoints. Then, $(X, d)$ is said to satisfy the $p$-Busemann curvature condition for some $p \in[1, \infty]$ if, for each $x, y, z, w \in X$, each midpoint $m(x, y) \in$ $X$ of $x$ and $y$ and each midpoint $m(z, w) \in X$ of $z$ and $w$, one has

$$
\begin{aligned}
& d(m(x, y), m(z, w)) \\
& \quad \leq\left(\frac{1}{2}\right)^{1 / p}\left(d^{p}(x, z)+d^{p}(y, w)\right)^{1 / p} .
\end{aligned}
$$

Assertion 6. Suppose a metric space $(X, d)$ which admits midpoints, with the midpoint map (or midset) $m: X \times$ $X \rightarrow X$ being unique, and which satisfies the $p$-Busemann curvature condition for some $p \in[1, \infty]$. Then, one has

$$
\begin{gathered}
d(m(x, y), m(z, w)) \leq\left(\frac{1}{2}\right)^{1 / p} \\
\cdot \min \left(\left(d^{p}(x, z)+d^{p}(y, w)\right)^{1 / p}\right. \\
\left.\left(d^{p}(x, w)+d^{p}(y, z)\right)^{1 / p}\right)
\end{gathered}
$$

for any $x, y, z, w \in X$, where $m(x, y) \in X$ and $m(z, w) \in X$ are, respectively, the unique midpoints of $x$ and $y$ and $w$ and $z$.

Proof. Since $(X, d)$ admits midpoints if $c=m(z, w)$, then the midpoint is unique since $m: X \times X \rightarrow X$ is unique [19], then $c=m(w, z)$. Thus, we can replace $m(z, w) \rightarrow m(w, z)$ leading to an alternative right-hand side in (7) under the replacements $z \rightarrow w$ and $w \rightarrow z$ which when combined with (7) leads to (8).
Since the right-hand side of (4) is an upper-bound of the right-hand side of (8), we get directly the following important result.

Assertion 7. Assume that a metric space $(X, d)$ is midpoint convex (then it is 1-convex from Assertion 2) with unique midpoint map and that it satisfies the $p$-Busemann curvature condition for some $p \in[1, \infty]$. Then, $(X, d)$ is $p$-convex.

The following technical definitions are of interest to characterize near-uniform convexity.

Definition 8 ( $\varepsilon$-separated family of points [16]). A family of points $\left(x_{i}\right)_{i \in I}$ is $\varepsilon$-separated if $\inf _{i \in I} d\left(x_{i}, x_{j}\right) \geq \varepsilon$.

Definition 9 (nearly uniformly convex space [16]). A $\infty$ convex metric space $(X, d)$ is said to be nearly uniformly convex if, for any $R>0$ and for any $\varepsilon$-separated infinite family $\left\{x_{i}\right\}_{i \in I}$, with $x_{i} \in X$, and any $y \in X$ such that $d\left(x_{i}, y\right) \leq$ $r \leq R, \forall i \in I$, there is some $\rho=\rho(\varepsilon, R)>0$ such that $B_{(1-\rho) r}(y) \cap c \ell\left(\operatorname{con}\left\{x_{i}\right\}_{i \in I}\right) \neq \varnothing$, where $c \ell\left(\operatorname{con}\left\{x_{i}\right\}_{i \in I}\right)$ is the closure of the convex hull of the family $\left\{x_{i}\right\}_{i \in I}$.

It turns out that $\infty$-convexity implies near-uniform convex but the converse is not true.

Definition 10 (see $[21,22])$. Let $(X, d)$ be a metric space. A mapping $W: X \times X \times[0,1] \rightarrow X$ is said to be a convex structure on $X$ if, for each $(x, y, t) \in X \times X \times[0,1]$ and $z \in X$,

$$
d(z, W(x, y, t)) \leq t d(z, x)+(1-t) d(z, y) .
$$

Definition 11 (see [21]). A convex metric space $(X, d, W)$ is said to be uniformly convex if, for any $\varepsilon>0$, there exists $\delta=$ $\delta(\varepsilon) \in(0,1]$ such that, for any $r>0$ and $x, y, z \in X$ with $\max (d(x, z), d(y, z)) \leq r$ and $d(x, y) \geq r \varepsilon$,

$$
d\left(z, W\left(x, y, \frac{1}{2}\right)\right) \leq r(1-\delta) .
$$

A uniformly convex metric space $(X, d, W)$ is also referred to commonly as uniformly 1-convex [16]. This concept may be generalized as follows.

Definition 12. A convex metric space $(X, d, W)$ is said to be uniformly $p$-convex if, for any $\varepsilon>0$, there exists $\delta=\delta(\varepsilon) \epsilon$ $(0,1]$ such that, for any $r>0$ and any $x, y, z \in X$ with $\max (d(x, z), d(y, z)) \leq r$ and $d(x, y) \geq r \varepsilon$,

$$
d\left(z, W\left(x, y, \frac{1}{2}\right)\right) \leq r(1-\delta)^{1 / p}
$$

Proposition 13. If a convex metric space $(X, d, W)$ is uniformly convex then it is uniformly $p$-convex for any $p \geq 1$.

Proof. Since $(X, d, W)$ is uniformly convex then, for any $\varepsilon_{1}>$ 0 , there exists $\delta_{1}=\delta\left(\varepsilon_{1}\right) \in(0,1)$ such that $(10)$ holds with $\delta=\delta_{1}$ any $r>0$ and any $x, y, z \in X$ subject to $\max (d(x, z), d(y, z)) \leq r$ and $d(x, y) \geq r \varepsilon_{1}$. Thus, $d(x, y) \geq$ $r \varepsilon_{1}=r^{p}\left(\varepsilon_{1} / r^{p-1}\right)$. Choosing $\varepsilon_{1}=\varepsilon_{p} r^{p-1}$ for any given $p \geq 1$ 
and any given arbitrary $\varepsilon_{p}>0$, it follows for $\delta_{p} \in[\max (1-$ $\left.\left.r^{1-p}\left(1-\delta_{1}\right), 0\right), 1\right)$ that $d^{p}(z, W(x, y, 1 / 2)) \leq r^{p}\left(1-\delta_{p}\right) \leq$ $r\left(1-\delta_{1}\right)$ so that $(X, d, W)$ is uniformly $p(\geq 1)$-convex.

Proposition 14. A convex metric space $(X, d, W)$ is nearly uniformly convex if, for any $\varepsilon>0$, there exists a strictly increasing $\delta=\delta(\varepsilon) \in[0,1]$ such that, for any $r>0$, any $z \in X$, and any $\mu=\varepsilon r$-separated infinite family $\left\{x_{i}\right\}_{i \in I} \subset X$ satisfying $\sup _{i \in I} d\left(x_{i}, z\right) \leq r$ and

$$
\begin{aligned}
d\left(y, W\left(x_{i}, x_{j}, \frac{1}{2}\right)\right) \leq & (1-\delta) ; \\
& \forall x_{i}, x_{j}\left(\neq x_{i}\right) \in\left\{x_{i}\right\}_{i \in I} \subset X
\end{aligned}
$$

for some $y \in \mathrm{B}_{(1-\delta) r}(z)$, where $\mathrm{B}_{\alpha}(z)$ denotes an open ball of radius acentred at $z$.

Proof. Set $z_{i j}=W\left(x_{i}, x_{j}, 1 / 2\right) \in X$ for any given $x_{i}, x_{j}(\neq$ $\left.x_{i}\right) \in\left\{x_{i}\right\}_{i \in I}$. Thus, if $K \subset X$, one has from (12) that

$$
d\left(y, c \ell\left(\operatorname{co} z_{i j}\right)\right) \leq d\left(z, z_{i j}\right) \leq r(1-\delta),
$$

since $z \in \bigcap_{i, j(\neq i)} c \ell\left(\operatorname{co}\left\{z_{i j}\right\}_{i, j(\neq i) \in I}\right)$ since $\sup _{i \in I} d\left(x_{i}, z\right) \leq r$ so that

$$
c \ell \mathrm{B}_{(1-\delta) r}(z) \cap c \ell\left(\operatorname{co}\left\{z_{i j}\right\}_{i, j(\neq i) \in I}\right) \neq \varnothing .
$$

Since $\mathrm{B}_{(1-\delta) r}(z) \supset\{y\}$ is trivially nonempty and $\operatorname{co}\left\{z_{i j}\right\}_{i, j(\neq i) \in I}=\operatorname{co}\left\{x_{i}\right\}_{i \in I}$ then $\mathrm{B}_{(1-\delta) r}(z) \cap c \ell\left(\operatorname{co}\left\{x_{i}\right\}_{i \in I}\right) \neq \varnothing$. Note also that, for any fixed $R \geq r, \delta=\delta(\varepsilon, R)=$ $\sup _{0<r \leq R} \delta(\varepsilon, r)$ exists fulfilling (12). From Definition 9, $(X, d, W)$ is nearly uniformly convex.

Note that $(X, d, W)$ is nearly uniformly convex; then it is not necessarily uniformly convex since it can happen that, for some $y(\neq z) \in \mathrm{B}_{(1-\delta) r}(z)$, any $\varepsilon r$-separated infinite family $\left\{x_{i}\right\}_{i \in I} \subset X$ satisfying $\sup _{i \in I} d\left(x_{i}, y\right) \leq r$ and all $\delta=\delta(\varepsilon) \epsilon$ $(0,1]$, there exists some $\delta_{0}=\delta_{0}(\varepsilon) \in(0,1]$ such that

$$
\begin{aligned}
& d\left(y, W\left(x_{i}, x_{j}, \frac{1}{2}\right)\right) \leq r\left(1-\delta_{0}\right) ; \\
& d\left(z, W\left(x_{i}, x_{j}, \frac{1}{2}\right)\right)>r(1-\delta) .
\end{aligned}
$$

However, the converse is true as reflected in the next result.

Proposition 15. If a convex metric space $(X, d, W)$ is uniformly convex, then it is nearly uniformly convex.

Proof. Note that if (10) is satisfied with $\sup _{i \in I} d\left(x_{i}, z\right) \leq r$, $\inf _{i \in I} d\left(x_{i}, y\right) \geq \varepsilon r$ for any given $z \in X$, then (12) is satisfied for $y=z \in \mathrm{B}_{(1-\delta) r}(z)$, the ball with center at such $z$ and radius $(1-\delta) r$.

Proposition 16. If $(X, d)$ is nearly uniformly convex and strictly $\infty$-convex then, for any $R>0$ and for any $\varepsilon$-separated infinite family $\left\{x_{i}\right\}_{i \in I}$, with $x_{i} \in X$, and any $y \in X$ such that $d\left(x_{i}, y\right) \leq r \leq R ; \forall i \in I$, there is $\rho_{r}=\rho_{r}\left(\varepsilon, \varepsilon_{1}, r\right) \in\left(0,1-\varepsilon_{1} / r\right]$ such that

$d\left(x_{i}, y\right) \geq \varepsilon_{1}=\inf _{x(\neq y) \in X} d(y, x)$,

$c \ell \mathrm{B}_{\varepsilon_{1}}(y) \cap c \ell\left(\operatorname{con}\left\{x_{i}\right\}_{i \in I}\right) \neq \varnothing$ and contains at most two points of $X$ such that $c \ell \mathrm{B}_{\varepsilon_{1}}(y) \cap c \ell\left(\operatorname{con}\left\{x_{i}\right\}_{i \in I}\right)=$ $\{w\}$ with $w \neq y$ if $y \notin\left\{x_{i}: i \in I\right\}$ and $c \ell \mathrm{B}_{\varepsilon_{1}}(y) \cap$ $c \ell\left(\operatorname{con}\left\{x_{i}\right\}_{i \in I}\right)=\{y, w\}$ if $y \in c \ell\left(\operatorname{con}\left\{x_{i}\right\}_{i \in I}\right)$ with $a$ choice $\rho_{r}=1-\varepsilon_{1} / r$.

Proof. Since $(X, d)$ is nearly uniformly convex and strictly $\infty$-convex then any nonempty closed convex subset of $X$ is a Chebyshev set [16]. Thus, for any $y \in X$, there are balls $B_{\varepsilon_{1}}(y)$ whose closures $c \ell B_{\varepsilon_{1}+\delta}(y) \supseteq\{y, w\}$ are Chebyshev sets consisting of at least two distinct points if their radius [16, 23] $\left(1-\rho_{r}\right) r=\varepsilon_{1}+\delta \geq \varepsilon_{1}=\inf _{x(\neq y) \in X} d(y, x)$ for any $\delta \geq 0$ with $\rho_{r}=\rho_{r}\left(\varepsilon, \varepsilon_{1}, r\right) \leq \rho=\rho(\varepsilon, R)$. Since Chebyshev sets have a unique nearest neighbor in $X$ for each $y \in X$, [24], one has for $\delta=0$ that $c \ell \mathrm{B}_{\varepsilon_{1}}(y)=\{y, w\}$ is a Chebyshev set of two unique elements one of them being its center $z$. Then, one has since $(X, d)$ is nearly uniformly convex that

$$
\begin{aligned}
& c \ell \mathrm{B}_{\left(1-\rho_{r}\right) r}(y) \cap c \ell\left(\operatorname{con}\left\{x_{i}\right\}_{i \in I}\right) \supset B_{\left(1-\rho_{r}\right) r}(y) \cap \\
& c \ell\left(\operatorname{con}\left\{x_{i}\right\}_{i \in I}\right) \neq\{\varnothing\} \text { for any } \rho_{r} \in\left(0,1-\varepsilon_{1} / r\right], \\
& B_{\varepsilon_{1}-\sigma}(y) \cap c \ell\left(\operatorname{con}\left\{x_{i}\right\}_{i \in I}\right)=\{\varnothing\} \text { for any } \sigma>0, \\
& c \ell \mathrm{B}_{\varepsilon_{1}}(y) \cap c \ell\left(\operatorname{con}\left\{x_{i}\right\}_{i \in I}\right)=\{w\} \text { with } w \neq y \text { if } y \notin \\
& c \ell\left(\operatorname{con}\left\{x_{i}\right\}_{i \in I}\right) \text { with } \rho_{r}=1-\varepsilon_{1} / r, \\
& c \ell \mathrm{B}_{\varepsilon_{1}}(y) \cap c \ell\left(\operatorname{con}\left\{x_{i}\right\}_{i \in I}\right)=\{y, w\} \text { if } y \in \\
& c \ell\left(\operatorname{con}\left\{x_{i}\right\}_{i \in I}\right) \text { with } \rho_{r}=1-\varepsilon_{1} / r .
\end{aligned}
$$

\section{Some Results on Contractiveness and Nonexpansiveness in CAT(0) Spaces}

A metric space $(X, d)$ is (uniquely) geodesic if every two points $x, y$ of $X$ are joined by a unique geodesic segment $[x, y]$ which is the image of the geodesic path from $x$ to $y$, that is, the isometry $c:[0, \ell] \rightarrow X$ such that $c(0)=x, c(\ell)=y$ and $\ell=d(x, y)$. A geodesic triangle $\Delta\left(x_{1}, x_{2}, x_{3}\right)$ consists of three vertices $x_{1}, x_{2}, x_{3} \in X$ and three geodesic segments joining each pair of vertices. A model comparison triangle $\bar{\Delta}\left(\bar{x}_{1}, \bar{x}_{2}, \bar{x}_{3}\right)$ of the geodesic triangle $\Delta\left(x_{1}, x_{2}, x_{3}\right)$ is a triangle in the Euclidean space $\mathbf{R}^{2}$ such that $d\left(x_{i}, x_{j}\right)=d_{\mathbf{R}^{2}}\left(\bar{x}_{i}, \bar{x}_{j}\right)$ for $i, j=1,2,3$. A geodesic metric space is a $\operatorname{CAT}(0)$ space $[4,16,21,22]$ if $d(x, y) \leq d_{\mathbf{R}^{2}}(\bar{x}, \bar{y}) ; \forall x, y \in \Delta, \forall \bar{x}, \bar{y} \in \bar{\Delta}$ (CAT(0) inequality). See, for instance, $[1,4-8]$.

In the paper, we write $(1-t) x \oplus t y$ for the unique $z_{t} \in X$ such that $d(z, x)=t d(x, y)$ and $d(z, y)=(1-t) d(x, y)$. Note that the midpoint of $[x, y]$ is $z_{1 / 2}=m(x, y)=(1 / 2) x \oplus(1 / 2) y$.

Note the following from the basic results in Section 2 .

(1) A geodesic space is a CAT(0) space if and only if for any $x, y, z \in X$ and all $t \in[0,1]$ the following inequality is satisfied:

$$
\begin{aligned}
d^{2}((1-t) x \oplus t y, z) \leq & (1-t) d^{2}(z, x)+t d^{2}(z, y) \\
& -t(1-t) d^{2}(x, y) .
\end{aligned}
$$

(Proposition $1.1[4])$. 
(2) A CAT(0) space is uniformly $p$-convex for any $p \geq 2$ [16].

(3) A CAT(0) space satisfies inequalities (4) and (5) for any $p \geq 2$ since it is midpoint $p(\geq 2)$-convex for any $p \geq 1$.

(4) $\operatorname{A~CAT(0)~space~satisfies~the~} p$-Busemann curvature condition for any $p \geq 1$ from (7).

A general technical result involving constructions with two self-mappings in a CAT(0) space as follows.

Lemma 17. Let a metric space $(X, d)$ be a CAT(0) space and let the mapping $Q_{t}: X \times X \rightarrow X$ be defined by

$$
Q_{t}(x, y)=t x \oplus(1-t) y ; \quad \forall t \in[0,1]
$$

for any $x, y \in X$ and let $T, S: X \rightarrow X$ be two self-mappings which satisfy the following conditions:

$$
\begin{aligned}
& d(T x, T y) \leq K_{T} d(x, y) \\
& d(S x, S y) \leq K_{S} d(x, y)
\end{aligned}
$$

for any given, some positive real constants $K_{T}$ and $K_{S}$. Then, for any given $x, y, p, q \in X$ and for any $t \in[0,1]$, the following properties hold:

(i)

$$
\begin{aligned}
& d^{2}\left(Q_{t}(S p, T x), Q_{t}(S q, T y)\right) \leq t^{2} K_{S}^{2} d^{2}(p, q)+(1 \\
& -t)^{2} K_{T}^{2} d^{2}(x, y)+t(1-t)(d(S p, T y)+d(S q, \\
& T y)+d(T x, S q)+d(S p, T x)) \min \left(K_{S} d(p, q),\right. \\
& \left.K_{T} d(x, y)\right) \\
& d^{2}\left(Q_{t}(S p, T x), Q_{t}(S q, T y)\right) \leq\left(t^{2} K_{S}^{2}+(1-t)^{2} K_{T}^{2}\right) \\
& \cdot \max \left(d^{2}(x, y), d^{2}(p, q)\right)+t(1-t)(d(S p, T y) \\
& +d(S q, T y)+d(T x, S q)+d(S p, T x)) \\
& \cdot \min \left(K_{S} d(p, q), K_{T} d(x, y)\right) \\
& d^{2}\left(Q_{t}(S p, T x), Q_{t}(S q, T y)\right) \leq\left(t^{2} K_{S}^{2}+(1-t)^{2} K_{T}^{2}\right. \\
& \left.+4 t(1-t) \min \left(K_{S}, K_{T}\right)\right) \times \max \left(d^{2}(x, y),\right. \\
& \quad d^{2}(p, q),
\end{aligned}
$$$$
(d(S p, T y), d(S q, T y), d(T x, S q), d(S p, T x))
$$$$
\cdot \min (d(p, q), d(x, y))) \text {. }
$$

$$
\begin{gathered}
d^{2}\left(Q_{t}\left(S^{n} p, T^{n} x\right), Q_{t}\left(S^{n} q, T^{n} y\right)\right) \leq t^{2} K_{S}^{2 n} d^{2}(x, y) \\
+(1-t)^{2} K_{T}^{2 n} d^{2}(p, q)+t(1-t)\left(d \left(S^{n-1} p,\right.\right.
\end{gathered}
$$

$$
\begin{aligned}
& \left.T^{n-1} y\right)+d\left(S^{n-1} q, T^{n-1} y\right)+d\left(T^{n-1} x, S^{n-1} q\right) \\
& \left.+d\left(S^{n-1} p, T^{n-1} x\right)\right) \times \min \left(K_{S} d\left(S^{n-1} p, S^{n-1} q\right)\right. \\
& \left.K_{T} d\left(T^{n-1} x, T^{n-1} y\right)\right) . \\
& d^{2}\left(Q_{t}\left(S^{n} p, T^{n} x\right), Q_{t}\left(S^{n} q, T^{n} y\right)\right) \leq\left(t^{2} K_{S}^{2 n}+(1\right. \\
& \left.-t)^{2} K_{T}^{2 n}\right) \max \left(d^{2}(x, y), d^{2}(p, q)\right)+t(1-t) \\
& \quad\left(d\left(S^{n-1} p, T^{n-1} y\right)+d\left(S^{n-1} q, T^{n-1} y\right)\right. \\
& \left.+d\left(T^{n-1} x, S^{n-1} q\right)+d\left(S^{n-1} p, T^{n-1} x\right)\right) d(p, q) \\
& \quad \times \min \left(K_{S}^{n}, K_{T}^{n}\right) \max (d(p, q), d(T x, T y)) \\
& d^{2}\left(Q_{t}\left(S^{n} p, T^{n} x\right), Q_{t}\left(S^{n} q, T^{n} y\right)\right) \leq\left(t^{2} K_{S}^{2 n}+(1\right. \\
& \left.-t)^{2} K_{T}^{2 n}+4 t(1-t) \min \left(K_{S}^{n}, K_{T}^{n}\right)\right) \\
& \quad \times \max \left(d^{2}(x, y), d^{2}(p, q), d\left(S^{n-1} p, T^{n-1} y\right),\right. \\
& d\left(S^{n-1} q, T^{n-1} y\right), d\left(T^{n-1} x, S^{n-1} q\right), \\
& \left.d\left(S^{n-1} p, T^{n-1} x\right)\right) \times \max (d(p, q), d(T x, T y))
\end{aligned}
$$

Proof. From (16) to (18), one gets:

$$
\begin{aligned}
d^{2} & \left(Q_{t}(S p, T x), Q_{t}(S q, T y)\right)=d^{2}(t S p \oplus(1-t) \\
\cdot & T x, t S q \oplus(1-t) T y) \leq t d^{2}(S p, t S q \oplus(1-t) T y) \\
+ & (1-t) d^{2}(T x, t S q \oplus(1-t) T y) \\
& -t(1-t) d^{2}(S p, T x) \leq t^{2} d^{2}(S p, S q) \\
+ & t(1-t) d^{2}(S p, T y)-t^{2}(1 \\
& -t) d^{2}(S q, T y)+t(1-t) d^{2}(T x, S q) \\
+ & (1-t)^{2} d^{2}(T x, T y)-t(1 \\
- & t)^{2} d^{2}(S q, T y)-t(1-t) d^{2}(S p, T x) \\
& =t^{2} d^{2}(S p, S q)+(1-t)^{2} d^{2}(T x, T y)+t(1-t) \\
& +\left(d^{2}(S p, T y)+d^{2}(T x, S q)\right) \\
& -\left(t(1-t)^{2}+t^{2}(1-t)\right) d^{2}(S q, T y) \\
& +t(1-t) d^{2}(S p, T x)=t^{2} d^{2}(S p, S q) \\
+ & (1-t)^{2} d^{2}(T x, T y)+t(1-t)
\end{aligned}
$$




$$
\begin{aligned}
& \cdot\left(d^{2}(S p, T y)+d^{2}(T x, S q)-d^{2}(S q, T y)\right. \\
& \left.-d^{2}(S p, T x)\right)
\end{aligned}
$$

On the other hand, one has by completion of squares that

$$
\begin{aligned}
& d^{2}(S p, T y)+d^{2}(T x, S q)-d^{2}(S q, T y) \\
& -d^{2}(S p, T x)=(d(S p, T y)+d(S q, T y)) \\
& \cdot(d(S p, T y)-d(S q, T y)) \\
& +(d(T x, S q)+d(S p, T x)) \\
& \cdot(d(T x, S q)-d(S p, T x)) \\
& d^{2}(S p, T y)+d^{2}(T x, S q)-d^{2}(S q, T y) \\
& -d^{2}(S p, T x)=(d(S p, T y)+d(S p, T x)) \\
& \cdot(d(S p, T y)-d(S p, T x)) \\
& +(d(T x, S q)+d(S q, T y)) \\
& \quad \cdot(d(T x, S q)-d(S q, T y))
\end{aligned}
$$

and from the triangle inequality for distances in (22a) and (22b) and the use of (18), one gets

$$
\begin{aligned}
& \max (d(S p, T y)-d(S q, T y), d(T x, S q) \\
& \quad-d(S p, T x)) \leq \min (d(S p, S q), d(T x, T y)) \\
& \quad \leq \min \left(K_{S} d(p, q), K_{T} d(x, y)\right) .
\end{aligned}
$$

The substitution of (23) into (22a) and (22b) with the use of (18) yields

$$
\begin{aligned}
& d^{2}(S p, T y)+d^{2}(T x, S q)-d^{2}(S q, T y)-d^{2}(S p, T x) \\
& \quad \leq(d(S p, T y)+d(T x, S q)+d(S q, T y) \\
& \quad+d(S p, T x)) \times \min \left(K_{S} d(p, q), K_{T} d(x, y)\right) .
\end{aligned}
$$

Now, the replacement of (18) and (24) into (21) leads to

$$
\begin{aligned}
& d^{2}\left(Q_{t}(S p, T x), Q_{t}(S q, T y)\right) \leq t^{2} K_{S}^{2} d^{2}(p, q)+(1 \\
& -t)^{2} K_{T}^{2} d^{2}(x, y)+t(1-t)(d(S p, T y) \\
& \quad+d(S q, T y)+d(T x, S q)+d(S p, T x)) \\
& \quad \cdot \min \left(K_{S} d(p, q), K_{T} d(x, y)\right) \leq\left(t^{2} K_{S}^{2}\right. \\
& \left.\quad+(1-t)^{2} K_{T}^{2}\right) \max \left(d^{2}(p, q), d^{2}(x, y)\right)+t(1 \\
& \quad-t)(d(S p, T y)+d(S q, T y)+d(T x, S q) \\
& \quad+d(S p, T x)) \min \left(K_{S} d(p, q), K_{T} d(x, y)\right)
\end{aligned}
$$

which implies (19a)-(19c) and the proof of Property (i) is complete. On the other hand, one has directly from (19a)-(19c) after replacing $p \rightarrow S^{n-1} p, q \rightarrow S^{n-1} q, x \rightarrow$ $S^{n-1} x, y \rightarrow S^{n-1} y$ for $n \in \mathbf{Z}_{+}$that (20a)-(20c) holds for any $n \in \mathbf{Z}_{+}$as counterpart of (19a)-(19c) and Property (ii) is proved.

From Lemma 17(ii), we get the following result.

Lemma 18. Let a metric space $(X, d)$ be a CAT(0) space and consider the mapping defined in (17) via two self-mappings $T, S: X \rightarrow X$ satisfying (18). Then, the following properties hold:

(i) Assume that $\max \left(K_{S}, K_{T}\right)<1$ (i.e., $S, T: X \rightarrow X$ are both strictly contractive). Then, $\lim _{n \rightarrow \infty} d\left(Q_{t}\left(S^{n} p, T^{n} x\right)\right.$, $\left.Q_{t}\left(S^{n} q, T^{n} y\right)\right)=0$ for any $x, y, p, q \in X$ and $t \in[0,1]$.

(ii) Assume that

(a) either $K_{S} \in(0,1)$ (i.e., $S: X \rightarrow X$ is strictly contractive), $K_{T}=1$ (i.e., $T: X \rightarrow X$ is nonexpansive but noncontractive), and $T: X \rightarrow X$ has a fixed point

(b) $K_{T} \in(0,1)$ (i.e., $T: X \rightarrow X$ is strictly contractive), $K_{S}=1$ (i.e., $S: X \rightarrow X$ is nonexpansive but noncontractive), and $S: X \rightarrow X$ has a fixed point.

Then,

$$
\begin{aligned}
& \underset{n \rightarrow \infty}{\limsup }\left[d^{2}\left(Q_{t}\left(S^{n} p, T^{n} x\right), Q_{t}\left(S^{n} q, T^{n} y\right)\right)\right. \\
& \left.\quad-\min \left(t^{2},(1-t)^{2}\right) \max \left(d^{2}(x, y), d^{2}(p, q)\right)\right] \\
& \quad \leq 0
\end{aligned}
$$

for any $x, y, p, q \in X$ and $t \in[0,1]$.

(iii) If $T, S: C \rightarrow C$ are both nonexpansive, where $C$ is a nonempty closed convex subset of $X$, then

$$
\begin{aligned}
& \limsup _{n \rightarrow \infty}\left[d^{2}\left(Q_{t}\left(S^{n} p, T^{n} x\right), Q_{t}\left(S^{n} q, T^{n} y\right)\right)\right. \\
& \quad-\left(t^{2}+(1-t)^{2}\right) \max \left(d^{2}(x, y), d^{2}(p, q)\right) \\
& \quad+t(1-t) M(p, q, x, y) \max (d(p, q), d(x, y))] \\
& \quad \leq 0
\end{aligned}
$$

for any $x, y, p, q \in X$ and $t \in[0,1]$.

Proof. It follows for constants $K_{S} \in(0,1)$ and $K_{T} \in(0,1)$ that both $T: X \rightarrow X$ and $T: X \rightarrow X$ are strict contractions on $X$ so that one gets from $(20 a)-(20 c)$ that

$$
\begin{aligned}
& \limsup _{n \rightarrow \infty}\left[d^{2}\left(Q_{t}\left(S^{n} p, T^{n} x\right), Q_{t}\left(S^{n} q, T^{n} y\right)\right)\right. \\
& \quad-t^{2} K_{S}^{2 n} d^{2}(x, y)-(1-t)^{2} K_{T}^{2 n} d^{2}(p, q)+t(1-t) \\
& \quad \cdot \min \left(K_{S}^{n}, K_{T}^{n}\right) \max (d(p, q), d(x, y)) \\
& \quad \times\left(d\left(S^{n-1} p, T^{n-1} y\right)+d\left(S^{n-1} q, T^{n-1} y\right)\right. \\
& \left.\left.\quad+d\left(T^{n-1} x, S^{n-1} q\right)+d\left(S^{n-1} p, T^{n-1} x\right)\right)\right] \leq 0 .
\end{aligned}
$$


Since $T: X \rightarrow X$ and $S: X \rightarrow X$ are strict contractions on $X$, $\left\{K_{S}^{n}\right\} \rightarrow 0$ and $\left\{K_{S T}^{n}\right\} \rightarrow 0$. Thus, $\exists \lim _{n \rightarrow \infty} d\left(Q_{t}\left(S^{n} p, T^{n} x\right)\right.$, $\left.Q_{t}\left(S^{n} q, T^{n} y\right)\right)=0$ for any $x, y, p, q \in X$ and $t \in[0,1]$. Property (i) has been proved.

Now, assume that either $K_{S} \in(0,1]$ and $K_{T}=1$ (then $T: X \rightarrow X$ has a fixed point since $(X, d)$ is a CAT( 0$)$ space, $[5,17,18])$, or $K_{T} \in(0,1]$ and $K_{S}=1$ (then $S: X \rightarrow X$ has a fixed point) and $t \in[0,1]$. Then, one has from (20a)-(20c) that $\left\{\min \left(K_{S}^{n}, K_{T}^{n}\right)\right\} \rightarrow 0$ :

$$
\begin{aligned}
& \limsup _{n \rightarrow \infty}\left[d^{2}\left(Q_{t}\left(S^{n} p, T^{n} x\right), Q_{t}\left(S^{n} q, T^{n} y\right)\right)\right. \\
& \quad-\min \left(t^{2},(1-t)^{2}\right) \\
& \quad \cdot \max \left(K_{S}^{2 n} d^{2}(x, y), K_{T}^{2 n} d^{2}(p, q)\right)+t(1-t) \\
& \quad \cdot \min \left(K_{S}^{n}, K_{T}^{n}\right) \max (d(p, q), d(x, y)) \\
& \quad \times\left(d\left(S^{n-1} p, T^{n-1} y\right)+d\left(S^{n-1} q, T^{n-1} y\right)\right. \\
& \left.\left.\quad+d\left(T^{n-1} x, S^{n-1} q\right)+d\left(S^{n-1} p, T^{n-1} x\right)\right)\right] \leq 0 .
\end{aligned}
$$

Assume with no loss in generality that $T: X \rightarrow X$ is strictly contractive $\left(K_{T}<1\right)$ with a unique fixed point $y^{*} \in X$ as a result, since $(X, d)$ is complete, and $S: X \rightarrow X$ is nonexpansive $\left(K_{S}=1\right)$ and has a fixed point $p^{*} \in X$ by hypothesis. Then,

$$
\begin{aligned}
& d\left(S^{n} p, T^{n} y\right) \leq d\left(S^{n} p, S^{n} p^{*}\right)+d\left(p^{*}, y^{*}\right) \\
&+d\left(T^{n} y^{*}, T^{n} y\right) \\
& \leq d\left(p, p^{*}\right)+d\left(p^{*}, y^{*}\right)+K_{T}^{n} d\left(y, y^{*}\right) \\
& \leq d\left(p, p^{*}\right)+d\left(p^{*}, y^{*}\right)+d\left(y, y^{*}\right) \\
&<\infty ; \quad \forall n \in \mathbf{Z}_{0+} \\
& d\left(S^{n} q, T^{n} y\right) \leq d\left(q, p^{*}\right)+d\left(p^{*}, y^{*}\right)+d\left(y, y^{*}\right) \\
&<\infty ; \quad \forall n \in \mathbf{Z}_{0+} \\
& d\left(S^{n} p, T^{n} x\right) \leq d\left(p, p^{*}\right)+d\left(p^{*}, y^{*}\right)+d\left(x, y^{*}\right) \\
&<\infty ; \quad \forall n \in \mathbf{Z}_{0+} \\
& d\left(S^{n} q, T^{n} x\right) \leq d\left(q, p^{*}\right)+d\left(p^{*}, y^{*}\right)+d\left(x, y^{*}\right) \\
&<\infty ; \quad \forall n \in \mathbf{Z}_{0+} .
\end{aligned}
$$

Thus, the sequence $\left\{d\left(S^{n} p, T^{n} y\right)+d\left(S^{n} q, T^{n} y\right)+d\left(T^{n} x, S^{n} q\right)+\right.$ $\left.d\left(S^{n} p, T^{n} x\right)\right\}$ is bounded for any given $p, q, x, y \in X$ for any distance $d: X \times X \rightarrow \mathbf{R}_{0+}$, and

$$
\left\{t(1-t) \min \left(K_{S}^{n}, K_{T}^{n}\right) \max (d(p, q), d(x, y))\right\}
$$

since $\left\{\min \left(K_{S}^{n}, K_{T}^{n}\right)\right\} \rightarrow 0$. Furthermore, since $K_{S}, K_{T} \in(0,1]$ with either $K_{S}=1$ or $K_{T}=1$, then one has from (28) that for $t \in[0,1]$ :

$$
\begin{aligned}
& \limsup _{n \rightarrow \infty}\left(K_{S}^{2 n} t^{2}+K_{T}^{2 n}(1-t)^{2}\right) \\
& \cdot \max \left(d^{2}(x, y), d^{2}(p, q)\right) \leq \min \left(t^{2},(1-t)^{2}\right) \\
& \cdot \max \left(d^{2}(x, y), d^{2}(p, q)\right) \\
& \quad \leq \max \left(d^{2}(x, y), d^{2}(p, q)\right),
\end{aligned}
$$

the one which is contractive has a unique fixed point since $(X, d)$ is complete; then $\left\{S^{n} z\right\},\left\{T^{n} w\right\},\left\{d\left(S^{n-1} p, T^{n-1} y\right)\right\}$ and then $\left\{d\left(S^{n-1} q, T^{n-1} y\right)\right\},\left\{d\left(T^{n-1} x, S^{n-1} q\right)\right\}$ and $\left\{d\left(S^{n-1} p\right.\right.$, $\left.\left.T^{n-1} x\right)\right\}$ are bounded sequences for any given $z, w, x, y, p, q \in$ $X$. Thus, one gets from (29)-(32) that (26) holds. Property (ii) has been proved.

Property (iii) follows since (27) holds from (28) with a finite real $M$ depending on the points $p, q, x, y$ of $X$.

$$
\begin{aligned}
M= & M(p, q, x, y) \\
\geq & d\left(S^{n} p, T^{n} y\right)+d\left(S^{n} q, T^{n} y\right)+d\left(T^{n} x, S^{n} q\right) \\
& +d\left(S^{n} p, T^{n} x\right) .
\end{aligned}
$$

Related to Lemma 18(ii), note that if $T, S: C \rightarrow C$ are both nonexpansive, where $C$ is a nonempty closed convex subset of $X$, and at least one of them is strictly contractive and the other one has a fixed point then (26) holds. This occurs despite that although a CAT(0) space is uniformly $p(\geq 2)$ convex [16], it can be nonuniformly convex, in general. However, the existence of (at least) a fixed point for the (noncontractive) expansive mapping $T$ or $S$ on $C$ is guaranteed $[5,17,18]$. The results below are concerned with sufficient conditions for the uniform convexity and near-uniform convexity of CAT( 0$)$ spaces.

Proposition 19. Let a geodesic metric space $(X, d, W)$ be a $C A T(0)$ space. Then, it is uniformly convex if, for any $\varepsilon \in[0,2]$, there exists a strictly increasing $\delta=\delta(\varepsilon) \in\left[0,1-\sqrt{2-\varepsilon^{2} / 4}\right]$, with $\delta(\varepsilon)>0$ for $\varepsilon>0$, such that $d(z, W(x, y, t)) \leq r(1-$ b) for $t \in[0,1]$, any $r>0$ and any $x, y, z \in X$ with $\max (d(x, z), d(y, z)) \leq r$ and $2 r \geq d(x, y) \geq r \varepsilon$.

Proof. A geodesic space is a uniformly convex CAT(0) space if, for any $t \in[0,1]$,

$$
\begin{aligned}
d^{2}((1-t) x \oplus t y, z) \leq & (1-t) d^{2}(z, x)+t d^{2}(z, y) \\
& -t(1-t) d^{2}(x, y) \\
\leq & 2 r^{2}-t(1-t) d^{2}(x, y) \\
\leq & \left(2-t(1-t) \varepsilon^{2}\right) r^{2} \\
\leq & r^{2}(1-\delta)^{2} ; \quad t \in[0,1]
\end{aligned}
$$


[4]. Note that (34) holds for any $x, y, z \in X$ fulfilling $\max (d(x, z), d(y, z)) \leq r$ and any $t \in[0,1]$ if

$$
\begin{aligned}
d^{2}(x, y) & \leq \min _{t \in[0,1]}\left(t^{-1}+(1-t)^{-1}\right) r^{2} \\
& =\frac{r^{2}}{\max _{t \in[0,1]}(t(t-1))}=4 r^{2} .
\end{aligned}
$$

For coherency of the above constraints with the distance properties for any given $x, y, z \in X$ with $\max (d(x, z)$, $d(y, z)) \leq r$ and $\varepsilon r \leq d(x, y) \leq 2 r$ for all $\varepsilon \in[0,2]$ subject to

$$
\begin{aligned}
0 & \leq \delta \leq 1-\max _{s \in[0,1]} \sqrt{2-s(1-s) \varepsilon^{2}}=1-\sqrt{2-\frac{\varepsilon^{2}}{4}} \\
& \leq 1-\sqrt{2-t(1-t) \varepsilon} ; \quad t \in[0,1]
\end{aligned}
$$

so that $\delta:[0,2] \rightarrow[0,1]$ satisfying $\delta=\delta(\varepsilon) \in[0,1-$ $\left.\sqrt{2-\varepsilon^{2} / 4}\right]$ is strictly increasing with $\delta(0)=0$ for any $\varepsilon \in$ $[0,2]$.

Proposition 20. Let a geodesic metric space $(X, d, W)$ be a uniformly convex CAT(O) space satisfying the conditions of Proposition 19. Then $(X, d, W)$ is nearly uniformly convex.

Proof. Since the CAT(0) space satisfies Proposition 19 then it is uniformly convex and uniformly $p(\geq 1)$ convex and then $\infty$-uniformly convex so it is nearly uniformly convex (Definition 9).

There are some particular results of Lemma 17 of interest concerning the role of just one of the two involved mappings. For instance, Lemma 17(i) yields directly the following result.

Theorem 21. Let the metric space $(X, d)$ be a CAT(0) space and consider the mapping defined in (17) via two self-mappings $T, S: C \rightarrow C$ satisfying (18), where $C$ is a nonempty closed convex subset of $X$. The following properties hold:

(i)

$$
\begin{array}{lr}
d\left(Q_{t}\left(S^{m} p, T x\right), Q_{t}\left(S^{m} p, T y\right)\right) \leq(1-t) & K_{T} d(x, y) ; \\
& \forall m \in \mathbf{Z}_{0+}, \\
d^{2}\left(Q_{t}\left(S^{m} p, T^{n} x\right), Q_{t}\left(S^{m} p, T^{n} y\right)\right) & \\
\leq(1-t) K_{T}^{n} d(x, y) ; \quad \forall n, m \in \mathbf{Z}_{0+}, & \forall m \in \mathbf{Z}_{0+}, \\
d\left(Q_{t}\left(S p, T^{m} x\right), Q_{t}\left(S q, T^{m} x\right)\right) \leq t K_{S} d(p, q) ; & \quad \forall n, m \in \mathbf{Z}_{0+} .
\end{array}
$$

(ii) If $(X, d)$ is complete then $Q_{t}: S^{m} p \times T C \rightarrow C$ is a strict contraction for each $t \in[0,1]$ and each $m \in \mathbf{Z}_{0+}$, irrespective of the mapping $S: C \rightarrow C$, for any given $p, q(=$ $p) \in X$ provided that $T: C \rightarrow C$ is strictly contractive. Thus,
$\left\{d\left(Q_{t}\left(S^{m} p, T^{n} x\right), Q_{t}\left(S^{m} p, T^{n} y\right)\right)\right\} \rightarrow 0, \forall m \in \mathbf{Z}_{0+}$ as $n \rightarrow \infty$, and has a unique fixed point:

$$
z=z_{t}\left(m, S, p, y^{*}\right)=t S^{m} p \otimes(1-t) y^{*}
$$

for each $t \in[0,1]$ and each given $p \in C$ and $m \in \mathbf{Z}_{0+}$ and the unique fixed point $y^{*}=T y^{*} \in C$ of $T: C \rightarrow C$ and $\left\{T^{n} y\right\} \rightarrow y^{*}$. In particular, if $t=0, z_{0}\left(y^{*}\right)=y^{*}$ and if $t=1$, $z_{1}\left(m, S, p, y^{*}\right)=S^{m} p$.

If both $S, T: C \rightarrow C$ are strictly contractive then $\left\{S^{m} p\right\} \rightarrow p^{*}=S p^{*}$ with Fix $S=\left\{p^{*}\right\}$ and then $z=$ $\lim _{m \rightarrow \infty} z_{t}\left(m, S, p^{*}, y^{*}\right)=t p^{*} \otimes(1-t) y^{*}$ for each $t \in[0,1]$ is the unique fixed point of $Q_{t}: S^{m} p \times T C \rightarrow C$ for each $t \in[0,1]$; $\forall m \in \mathbf{Z}_{0+}$.

If $T: C \rightarrow C$ is strictly contractive and $S: C \rightarrow C$ is nonexpansive with $p^{*} \in$ Fix $S$ (Fix $S=\left\{p^{*}\right\}$ if $S: C \rightarrow C$ is strictly contractive) then $Q_{t}: S^{m} p^{*} \times T C \rightarrow C$ has a unique fixed point $z_{t}=t p^{*} \otimes(1-t) y^{*}$ for each $t \in[0,1] ; \forall m \in \mathbf{Z}_{0+}$.

(iii) If $(X, d)$ is complete then $Q_{t}: X \times X \rightarrow X$ is a strict contraction, irrespective of the mapping $T: C \rightarrow C$, for any given $x, y(=x) \in C$ provided that $S: C \rightarrow C$ is strictly contractive so that it has a unique fixed point and then $\left\{d\left(Q_{t}\left(S^{n} p, T^{m} x\right), Q_{t}\left(S^{n} p, T^{m} y\right)\right)\right\} \rightarrow 0 ; \forall m \in \mathbf{Z}_{0+}$ as $n \rightarrow \infty$ and has a unique fixed point:

$$
z=z_{t}\left(m, T, p^{*}, x\right)=t p^{*} \otimes(1-t) T^{m} x
$$

for each $t \in[0,1]$ and each given $x \in C$ and $m \in \mathbf{Z}_{0+}$ and the unique existing fixed point $p^{*}=S p^{*} \in C$ of the nonexpansive mapping $S: C \rightarrow C$.

Proof. Equations (37) follow directly from ((19a)-(19c) and (20a)-(20c)) and Property (i) is proved directly. If, in addition, the CAT(0) space is a complete; then if $T: C \rightarrow C$ is strictly contractive, then and $C$ is nonempty, closed, and convex; $T: C \rightarrow C$ has a unique fixed point $y^{*} \in C$, $\left\{T^{n} x\right\} \rightarrow y^{*}$ for any $x \in C$ and $\left\{S^{n} p\right\}$ and $\left\{T^{n} x\right\}$ are bounded for any $x, p \in C$ since $C$ is bounded. From the second inequality of (37), $\left\{Q_{t}\left(S^{m} p, T^{n} x\right)-Q_{t}\left(S^{m} p, T^{n} y\right)\right\} \rightarrow 0$ as $n \rightarrow \infty, \forall m \in \mathbf{Z}_{0+}$ for all $t \in[0,1]$. Then, $\left\{Q_{t}\left(S^{m} p, T^{n} x\right)\right\}$ is a Cauchy sequence with a unique limit $z=z_{t}\left(m, S, p, y^{*}\right)=$ $t S^{m} p \otimes(1-t) y^{*}$ for each $t \in[0,1]$ and each $m \in \mathbf{Z}_{0+}$. Note that, for each $p \in C, z=z_{t}\left(m, S, p, y^{*}\right)$ is a unique fixed point of $Q_{t}: S^{m} p \times T C \rightarrow C$ since

$$
\begin{aligned}
Q_{t}\left(S^{m} p, T y^{*}\right) & =t S^{m} p \oplus(1-t) T y^{*} \\
& =t S^{m} p \oplus(1-t) y^{*}=Q_{t}\left(S^{m} p, y^{*}\right) .
\end{aligned}
$$

The corresponding results for the case that $S$ is also contractive or nonexpansive are direct counterparts of the above reasoning. Property (ii) has been proved. The proof of Property (iii) is similar and then it is omitted.

Different classes of iterative schemes and their stability properties related to fixed point theory as Halpern, Jungck, Ishikawa, and many of its variants and extensions have been studied in a number of papers. See, for instance, $[1,2,4,5$, $25,25-28$ ] and some references therein. The following result links an iterative scheme based on two maps to the convergence properties in $\mathrm{CAT}(0)$ spaces. 
Theorem 22. Let the metric space $(X, d)$ be a CAT(0) space and consider the iterative scheme:

$$
\begin{array}{r}
x_{n+2}=Q_{t_{n}}\left(S_{n} x_{n+1}, T_{n} x_{n}\right)=t_{n} S_{n} x_{n+1} \oplus\left(1-t_{n}\right) T_{n} x_{n} ; \\
\forall n \in \mathbf{Z}_{0+}
\end{array}
$$

subject to any initial conditions $x_{0}, x_{1} \in X$ and define

$$
\theta_{n+1}=\frac{d\left(S_{n} x_{n+2}, T_{n} x_{n}\right)+d\left(S_{n} x_{n+1}, T_{n} x_{n}\right)+d\left(T_{n} x_{n+1}, S_{n} x_{n+1}\right)+d\left(S_{n} x_{n+2}, T_{n} x_{n+1}\right)}{\max \left(d\left(x_{n+1}, x_{n+2}\right), d\left(x_{n}, x_{n+1}\right)\right)} ; \quad \forall n \in \mathbf{Z}_{0+}
$$

with $\left\{\theta_{n}\right\}$ being dependent, in general, on $x_{0}$ and $x_{1}$.

Then, the following properties hold:

(i) Assume that $\sup _{n \in \mathbf{Z}_{0+}} \theta_{n} \leq \theta<+\infty$ with $\theta$ being dependent, in general, on $x_{0}$ and $x_{1}$. Then

(a) if $\left\{T_{n}\right\}$ is a sequence of nonexpansive (resp., strictly contractive) sequence of mappings then there is a real sequence $\left\{t_{n}\right\} \subset[0,1]$ of sufficiently small elements such that $\left\{\rho_{n}\right\} \subset[0,1]$ (resp., $\left\{\rho_{n}\right\} \subset[0,1)$ ),

(b) if $\left\{S_{n}\right\}$ is a sequence of nonexpansive (resp., strictly contractive) sequence of mappings then there is a real sequence $\left\{t_{n}\right\} \subset[0,1]$ of elements being sufficiently close to unity such that $\left\{\rho_{n}\right\} \subset[0,1]$ (resp., $\left\{\rho_{n}\right\} \subset$ $[0,1))$.

(ii)

$$
\begin{aligned}
& d^{2}\left(x_{n+2}, x_{n+3}\right) \\
& \quad \leq \rho_{n+1} \max \left(d^{2}\left(x_{n+1}, x_{n+2}\right), d^{2}\left(x_{n}, x_{n+1}\right)\right)
\end{aligned}
$$

$\forall n \in \mathbf{Z}_{0+}$,

where

$$
\begin{aligned}
\rho_{n+1}= & t_{n+1}^{2} K_{S_{n+1}}^{2}+\left(1-t_{n+1}\right)^{2} K_{T_{n+1}}^{2} \\
& +t_{n+1}\left(1-t_{n+1}\right) \min \left(K_{S_{n+1}}, K_{T_{n+1}}\right) \theta_{n+1} ; \\
& \forall n \in \mathbf{Z}_{0+}
\end{aligned}
$$

for any $t_{n} \in[0,1] ; \forall n \in \mathbf{Z}_{0+}$, and

$$
\begin{aligned}
& d^{2}\left(x_{n+m_{n}}, x_{n+m_{n}+1}\right) \leq \rho_{n+m_{n}-1} \\
& \cdot \max \left(d^{2}\left(x_{n+m_{n}-1}, x_{n+m_{n}}\right), d^{2}\left(x_{n+m_{n}-2}, x_{n+m_{n}-1}\right)\right) \\
& \quad \leq\left(\prod_{i=0}^{m_{n}-1}\left[\rho_{n+i}\right]\right)
\end{aligned}
$$

$$
\cdot \max \left(d^{2}\left(x_{n+1}, x_{n+2}\right), d^{2}\left(x_{n}, x_{n+1}\right)\right) ;
$$$$
m_{n} \geq 2, \forall n \in \mathbf{Z}_{0+} \text {. }
$$

(iii) If $x_{0}, x_{1} \in X$ and $x_{2}=Q_{t 0}\left(S x_{1}, T x_{0}\right)$ are such that $d\left(x_{1}, x_{2}\right) \leq d\left(x_{0}, x_{1}\right)$ and $\rho_{n} \leq 1, \forall n \in \mathbf{Z}_{0+}$, then

$$
d\left(x_{n+2}, x_{n+3}\right) ; \quad \forall n \in \mathbf{Z}_{0+} .
$$

The above inequality is strict if $\rho_{n}<1, \forall n \in \mathbf{Z}_{0+}$.

(iv) If there is a sequence $\left\{m_{n}\right\}$ of integers satisfying $2 \leq$ $m_{a} \leq m_{n} \leq m_{b}<\infty, \forall n \in \mathbf{Z}_{0+}$, such that $\prod_{i=0}^{m_{n}-1}\left[\rho_{n+i}\right] \leq \rho<$ 1 then $\lim _{n \rightarrow \infty} d\left(x_{n}, x_{n+1}\right)=\lim _{n \rightarrow \infty} d\left(x_{n+m_{n}}, x_{n+m_{n}+1}\right)=0$.

(v) Define the following sequences:

$$
\begin{aligned}
\alpha_{n} & =t_{n}^{2} K_{S_{n}}^{2}+\left(1-t_{n}\right)^{2} K_{T_{n}}^{2} \\
\beta_{n} & =t_{n}\left(1-t_{n}\right) \min \left(K_{S_{n}}, K_{T_{n}}\right)\left(d\left(S_{n} x_{n+1}, T_{n} x_{n-1}\right)\right. \\
& +d\left(S_{n} x_{n}, T_{n} x_{n-1}\right)+d\left(T_{n} x_{n}, S_{n} x\right) \\
& \left.+d\left(S_{n} x_{n+1}, T_{n} x_{n}\right)\right) ; \quad \forall n \in \mathbf{Z}_{+} .
\end{aligned}
$$

If $\left(\alpha_{n}+\beta_{n}\right)<1, \forall n \in \mathbf{Z}_{+}$, then $d\left(x_{n+1}, x_{n}\right) \rightarrow 0$ as $n \rightarrow \infty$. If, in addition, $(X, d)$ is a complete CAT( 0$)$ space then (1) $\left\{x_{n}\right\}$ converges to a limit $x^{*} \in X$ which is unique if $t_{n}=t \in[0,1]$, $\forall n \in \mathbf{Z}_{0+}$. (2) If $\left\{t_{n}\right\}(\subset[0,1)) \rightarrow t \in[0,1]$ and the sequence of mappings $\left\{Q_{t_{n}}\right\}$ converges point-wise to $Q_{t}$, then $Q_{t}$ is a strict contraction and $\left\{x_{n}^{*}\right\} \rightarrow x^{*}$, where $\operatorname{Fix}\left(Q_{t_{n}}\right)=\left\{x_{n}^{*}\right\}$, $\forall n \in \mathbf{Z}_{0+}$, and $x^{*} \in \operatorname{Fix}\left(T^{*}\right)$. (3) If $\left\{t_{n}\right\}(\subset[0,1)) \rightarrow t \in[0,1]$ and the sequence of mappings $\left\{Q_{t_{n}}\right\}$ with $\operatorname{Fix}\left(Q_{t_{n}}\right)=\left\{x_{n}^{*}\right\}$; $\forall n \in \mathbf{Z}_{0+}$ converges uniformly to a strict contraction $Q_{t}$ with $\operatorname{Fix}\left(Q_{t}\right)=\left\{x^{*}\right\}$, then $\left\{x_{n}^{*}\right\} \rightarrow x^{*}$.

(vi) Property (v) also holds if the constraint $\left(\alpha_{n}+\beta_{n}\right)<1$, $\forall n \in \mathbf{Z}_{+}$, is replaced with $\rho_{n}<1 ; \forall n \in \mathbf{Z}_{+}$.

Proof. Note that, for any sequence $\left\{K_{T_{n}}\right\} \subset[0,1]$, a sequence $\left\{\bar{t}_{n}\right\} \subset[0,1]$ exists such that if $\sup _{n \in \mathbf{Z}_{0+}} \theta_{n} \leq \theta<+\infty, t_{n} \in$ $\left[0, \bar{t}_{n}\right] ; \forall n \in \mathbf{Z}_{0+},\left\{\rho_{n}\right\} \subset[0,1]$ since 


$$
\begin{aligned}
\rho_{n} & =\rho_{n}\left(t_{n}\right)=t_{n}^{2} K_{S_{n}}^{2}+\left(1-t_{n}\right)^{2} K_{T_{n}}^{2}+t_{n}\left(1-t_{n}\right) \min \left(K_{S_{n}}, K_{T_{n}}\right) \theta_{n} \\
& =t_{n}\left(1-t_{n}\right)\left(\min \left(K_{S_{n}}, K_{T_{n}}\right) \frac{d\left(S_{n} x_{n+2}, T_{n} x_{n}\right)+d\left(S_{n} x_{n+1}, T x_{n}\right)+d\left(T_{n} x_{n+1}, S_{n} x_{n+1}\right)+d\left(S_{n} x_{n+2}, T_{n} x_{n+1}\right)}{\max \left(d\left(x_{n+1}, x_{n+2}\right), d\left(x_{n}, x_{n+1}\right)\right)}\right) \\
& +\left(t_{n}-1\right)^{2} K_{T_{n}}^{2}+t_{n}^{2} K_{S_{n}}^{2} \leq 1 ; \quad \forall n \in \mathbf{Z}_{0+} .
\end{aligned}
$$

Note that $\rho_{n}(t)$ is a continuous real function on $\mathbf{R}_{0+}$ with $\rho_{n}(0)=K_{T_{n}}^{2} \leq 1$ (if $K_{T_{n}}^{2} \leq 1$ ), that is, if $\left\{T_{n}\right\}$ is a nonexpansive sequence of mappings, and $\rho_{n}(0)<1$ (if $K_{T_{n}}^{2}<1$ ); $\forall n \in \mathbf{Z}_{0+}$ so that there is a sequence $\left\{\bar{t}_{n}\right\} \subseteq[0, \varepsilon] \subseteq[0,1]$ such that $\rho_{n}\left(t_{n}\right) \leq 1$ if $t_{n} \in\left[0, \bar{t}_{n}\right] ; \forall n \in \mathbf{Z}_{0+},\left\{K_{T_{n}}\right\} \subset[0,1], \rho_{n}\left(t_{n}\right)=$ $K_{T_{n}}^{2}<1$ and $\rho_{n}\left(t_{n}\right)<1$ if $\left\{\bar{t}_{n}\right\} \subset[0,1)$ and $\left\{K_{T_{n}}\right\} \subset[0,1)$, that is, if $\left\{T_{n}\right\}$ is a strictly contractive sequence. The same conclusions arise for $\left\{S_{n}\right\}$ being a nonexpansive sequence of mappings, respectively, a strictly contractive sequence (i.e., $\left\{K_{S_{n}}\right\} \subset[0,1]$, resp., $\left.\left\{K_{S_{n}}\right\} \subset[0,1)\right)$ if $\left\{\bar{t}_{n}\right\} \subseteq[1-\varepsilon, 1] \subseteq[0,1]$, respectively, $\left\{\bar{t}_{n}\right\} \subset[0,1)$ since $\rho_{n}(0)=K_{S_{n}}^{2} \leq 1$ (if $K_{S_{n}}^{2} \leq 1$ ) and $\rho_{n}(0)=K_{S_{n}}^{2}<1$ (if $K_{S_{n}}^{2}<1$ ). Property (i) has been proved.

Property (ii) follows by direct calculation from (19a)-(19c). On the other hand, if $d\left(x_{1}, x_{2}\right) \leq d\left(x_{0}, x_{1}\right)$ proceed by complete induction and contradiction by assuming that $d\left(x_{j+2}, x_{j+3}\right) \leq d\left(x_{j}, x_{j+1}\right) ; \forall j(<n) \in \mathbf{Z}_{0+}$ and some given $n(>2) \in \mathbf{Z}_{+}$and that $d\left(x_{n+2}, x_{n+3}\right)>d\left(x_{n}, x_{n+1}\right)$. Thus,

$$
\begin{aligned}
d^{2}\left(x_{n}, x_{n+1}\right) & <d^{2}\left(x_{n+2}, x_{n+3}\right) \\
& \leq \max \left(d^{2}\left(x_{n+1}, x_{n+2}\right), d^{2}\left(x_{n}, x_{n+1}\right)\right) \\
& \leq d^{2}\left(x_{n}, x_{n+1}\right) \quad \forall n \in \mathbf{Z}_{0+} .
\end{aligned}
$$

Then, $d\left(x_{n+2}, x_{n+3}\right) \leq d\left(x_{n}, x_{n+1}\right) ; \forall n \in \mathbf{Z}_{0+}$ if $\rho_{n} \leq 1$ and $d\left(x_{j+2}, x_{j+3}\right) \leq d\left(x_{j}, x_{j+1}\right) ; \forall j(<n) \in \mathbf{Z}_{0+} ; \forall n \in \mathbf{Z}_{0+}$ and Property (iii) is proved. If $\rho_{n}<1 ; \forall n \in \mathbf{Z}_{0+}$, then the relevant inequalities by reasoning by contradiction become

$$
\begin{aligned}
d^{2}\left(x_{n}, x_{n+1}\right) & \leq d^{2}\left(x_{n+2}, x_{n+3}\right) \\
& <\max \left(d^{2}\left(x_{n+1}, x_{n+2}\right), d^{2}\left(x_{n}, x_{n+1}\right)\right) \\
& \leq d^{2}\left(x_{n}, x_{n+1}\right) ; \quad \forall n \in \mathbf{Z}_{0+},
\end{aligned}
$$

again a contradiction. Then, $d\left(x_{n+2}, x_{n+3}\right)<d\left(x_{n}, x_{n+1}\right)$ if $\rho_{n}<1 ; \forall n \in \mathbf{Z}_{0+}$.

From the fact that $\prod_{i=0}^{m_{n}-2}\left[\rho_{n+i}\right] \leq \rho<1$, one gets that $\lim _{n \rightarrow \infty} d\left(x_{n}, x_{n+1}\right)=\lim _{n \rightarrow \infty} d\left(x_{n+m_{n}}, x_{n+m_{n}+1}\right)=0$ so that it follows by combining the above result with those of Properties (ii)-(iii) that Property (iv) is proved.
To prove Property (v), first note that

$$
\begin{aligned}
& d^{2}\left(x_{n+2}, x_{n+3}\right) \\
& \leq \alpha_{n} \max \left(d^{2}\left(x_{n+1}, x_{n+2}\right), d^{2}\left(x_{n}, x_{n+1}\right)\right) \\
& \quad+\beta_{n+1} \max \left(d^{2}\left(x_{n+1}, x_{n+2}\right), d^{2}\left(x_{n}, x_{n+1}\right)\right) .
\end{aligned}
$$

Case 1. $\max \left(d^{2}\left(x_{n+1}, x_{n+2}\right), d^{2}\left(x_{n}, x_{n+1}\right)\right) \geq 1$ so that

$$
\begin{aligned}
& d^{2}\left(x_{n+2}, x_{n+3}\right) \leq\left(\alpha_{n+1}+\beta_{n+1}\right) \\
& \cdot \max \left(d^{2}\left(x_{n+1}, x_{n+2}\right), d^{2}\left(x_{n}, x_{n+1}\right)\right) .
\end{aligned}
$$

Case 2. $\max \left(d^{2}\left(x_{n+1}, x_{n+2}\right), d^{2}\left(x_{n}, x_{n+1}\right)\right) \leq 1$ so that

$$
\begin{aligned}
& d^{2}\left(x_{n+2}, x_{n+3}\right) \\
& \quad \leq\left(\alpha_{n+1}+\beta_{n+1}\right) \max \left(d\left(x_{n+1}, x_{n+2}\right), d\left(x_{n}, x_{n+1}\right)\right) .
\end{aligned}
$$

Assume that Case 1 holds. Then since $\left(\alpha_{n}+\beta_{n}\right)<$ 1 and $\max \left(d^{2}\left(x_{n+1}, x_{n+2}\right), d^{2}\left(x_{n}, x_{n+1}\right)\right) \geq 1 ; \forall n \in$ $\mathbf{Z}_{+}$then $\lim \sup _{n \rightarrow \infty} d^{2}\left(x_{n+2}, x_{n+3}\right) \leq 1$. Otherwise, if $\lim \sup _{n \rightarrow \infty} d^{2}\left(x_{n+2}, x_{n+3}\right)>1$ then there is a subsequence $\left\{d^{2}\left(x_{n_{k}+2}, x_{n_{k}+3}\right)\right\}$ such that $d^{2}\left(x_{n_{k}+2}, x_{n_{k}+3}\right)>1$ for the subsequence $\left\{n_{k}\right\} \subset \mathbf{Z}_{0+}, k \in \mathbf{Z}_{0+}$ and, since $\left(\alpha_{n}+\beta_{n}\right)<1 ; \forall n \in \mathbf{Z}_{+}$, we get the contradiction $0 \geq \lim \sup _{k \rightarrow \infty} d^{2}\left(x_{n_{k}+2}, x_{n_{k}+3}\right)>1$. Thus, $\lim \sup _{n \rightarrow \infty} d^{2}\left(x_{n+2}, x_{n+3}\right) \leq 1$.

Now, if $\lim _{\sup _{n \rightarrow \infty}} d^{2}\left(x_{n+2}, x_{n+3}\right)=1$ which implies $\lim _{n \rightarrow \infty} d^{2}\left(x_{n+2}, x_{n+3}\right)=1$ this contradicts the contractive property associated with the condition $\left(\alpha_{n}+\beta_{n}\right)<1 ; \forall n \in \mathbf{Z}_{+}$. So, $\lim \sup _{n \rightarrow \infty} d^{2}\left(x_{n+2}, x_{n+3}\right)<1$. Now, Case 2 applies and two situations can arise for each $n \in \mathbf{Z}_{+}$, namely, either

$$
\begin{aligned}
& d^{2}\left(x_{n+2}, x_{n+3}\right) \leq d\left(x_{n+2}, x_{n+3}\right) \\
& \quad \leq\left(\alpha_{n+1}+\beta_{n+1}\right) \max \left(d\left(x_{n+1}, x_{n+2}\right), d\left(x_{n}, x_{n+1}\right)\right)
\end{aligned}
$$

or

$$
\begin{aligned}
& d\left(x_{n+2}, x_{n+3}\right) \geq \max \left(\left(\alpha_{n+1}+\beta_{n+1}\right)\right. \\
& \cdot \max \left(d\left(x_{n+1}, x_{n+2}\right), d\left(x_{n}, x_{n+1}\right)\right) \\
& \left.d^{2}\left(x_{n+2}, x_{n+3}\right)\right)=\left(\alpha_{n+1}+\beta_{n+1}\right) \\
& \cdot \max \left(d\left(x_{n+1}, x_{n+2}\right), d\left(x_{n}, x_{n+1}\right)\right) \geq d^{2}\left(x_{n+2},\right. \\
& \left.x_{n+3}\right) .
\end{aligned}
$$


Assume that there is a subsequence $\left\{d\left(x_{n_{k}+2}, x_{n_{k}+3}\right)\right\}$ which does not converge to zero. Then, the whole sequence $\left\{d\left(x_{n+2}, x_{n+3}\right)\right\}$ does not converge to zero. If $\left\{d\left(x_{n_{k}+2}, x_{n_{k}+3}\right)\right\}$ is bounded, we get from (55) the following contradiction:

$$
\begin{aligned}
0 & <\liminf _{m \rightarrow \infty} d\left(x_{n+m+2}, x_{n+m+3}\right) \\
& \geq \lim _{m \rightarrow \infty}\left(\prod_{j=1}^{m}\left(\alpha_{n+j}+\beta_{n+j}\right)\right) \\
& \cdot \max \left(d\left(x_{n+1}, x_{n+2}\right), d\left(x_{n}, x_{n+1}\right)\right)=0 \\
& \geq \liminf _{m \rightarrow \infty} d^{2}\left(x_{n+m+2}, x_{n+m+3}\right) .
\end{aligned}
$$

Thus, $\left\{d\left(x_{n_{k}+2}, x_{n_{k}+3}\right)\right\}$ cannot be bounded if it does not converge to zero so that it is unbounded. But, in Case 2, the distance is bounded since it is less than unity. As a result, $d\left(x_{n+1}, x_{n}\right) \rightarrow 0$ as $n \rightarrow \infty$ and $\left\{x_{n}\right\}$ converges to a unique limit if the CAT(0) metric space is complete if $t_{n}=t \in$ $[0,1]$ since then $Q_{t}$ is a strict contraction on $X$ of constant $\sup _{n \in \mathbf{Z}_{0+}}\left(\alpha_{n}+b_{n}\right)<1$. If $\left\{t_{n}\right\}(\subset[0,1]) \rightarrow t \in[0,1]$ and the sequence of (strictly) contractive mappings $\left\{Q_{t_{n}}\right\}$ (i.e., $\left.\sup _{n \in \mathbf{Z}_{0+}}\left(\alpha_{n}+b_{n}\right)<1\right)$ converges point-wise to $Q_{t}$ then $Q_{t}$ is a strict contraction and the sequence $\left\{x_{n}^{*}\right\} \rightarrow x^{*}$, where $x_{n}^{*} \in \operatorname{Fix}\left(Q_{t_{n}}\right)$ is unique; $\forall n \in \mathbf{Z}_{0+}$ and $x^{*} \in \operatorname{Fix}\left(T^{*}\right)$. If $t_{n}(\in[0,1]) \rightarrow t, x_{n}^{*} \in \operatorname{Fix}\left(Q_{t_{n}}\right), \forall n \in \mathbf{Z}_{0+},\left\{Q_{t_{n}}\right\} \rightarrow Q_{t}$ uniformly, where $Q_{t}$ is a strict contraction with $\operatorname{Fix}\left(Q_{t}\right)=x^{*}$, then there is a subsequence $\left\{Q_{t_{n_{k}}}\right\}$ of $\left\{Q_{t_{n}}\right\}$ of mappings on $X\left\{Q_{t_{n_{k}}}\right\} \rightarrow Q_{t}$ (being convergent strict contractions to $Q_{t}$ since $Q_{t}$ is a strict contraction) such that $\operatorname{Fix}\left(Q_{t_{n_{k}}}\right)=\left\{x_{n_{k}}^{*}\right\}$, $\forall k \in \mathbf{Z}_{0+}$ and $\left\{x_{n_{k}}^{*}\right\} \rightarrow x^{*}[27,28]$. Property (v) has been proved. It is evident from Properties (ii) and (v) that Property (vi) also holds if the constraint $\left(\alpha_{n}+\beta_{n}\right)<1 ; \forall n \in \mathbf{Z}_{+}$is replaced with $\rho_{n}<1 ; \forall n \in \mathbf{Z}_{+}$.

Example 23. Assume that $S_{n}=T_{n} ; \forall n \in \mathbf{Z}_{0+}$. Then,

$$
\begin{aligned}
& d\left(S_{n} x_{n+2}, T_{n} x_{n}\right)+d\left(S_{n} x_{n+1}, T_{n} x_{n}\right) \\
& \quad+d\left(T_{n} x_{n+1}, S_{n} x_{n+1}\right)+d\left(S_{n} x_{n+2}, T_{n} x_{n+1}\right) \\
& \quad \leq 2\left(d\left(T_{n} x_{n+2}, T_{n} x_{n+1}\right)+d\left(T_{n} x_{n+1}, T_{n} x_{n}\right)\right) \\
& \quad \leq 2 K_{T}\left(d\left(x_{n+2}, x_{n+1}\right)+d\left(x_{n+1}, x_{n}\right)\right) \\
& \quad \leq 4 K_{T_{n}} \max \left(d\left(x_{n+2}, x_{n+1}\right), d\left(x_{n+1}, x_{n}\right)\right)
\end{aligned}
$$

$\forall n \in \mathbf{Z}_{0+}$.

Thus, $\rho_{n}=\left(1+2 t_{n}\left(1-t_{n}\right)\right) K_{T_{n}}^{2}<1 ; \forall n \in \mathbf{Z}_{0+}$ if $q\left(t_{n}, K_{T_{n}}\right)=$ $1-\rho_{n}=1-\left(1+2 t_{n}\left(1-t_{n}\right)\right) K_{T_{n}}^{2}>0 ; \forall n \in \mathbf{Z}_{0+}$. Since $q\left(t_{n}, K_{T_{n}}\right)=0$ if $t_{n_{1,2}}=1 \pm \sqrt{2-K_{T_{n}}^{-2}}$ for each given $n \in \mathbf{Z}_{0+}$ then $q\left(t_{n}, K_{T_{n}}\right)>0$ and $t_{n} \in[0,1]$ if $t_{n_{1,2}}$ have nonzero imaginary values $\left(K_{T_{n}} \in[0,1 / \sqrt{2})\right)$ or if they are real $\left(K_{T_{n}} \in[1 / \sqrt{2}, 1)\right)$ and $t_{n} \in\left[0, t_{n_{1}}\right] \cup\left[t_{n_{2}}, 1\right] ; \forall n \in \mathbf{Z}_{0+}$. Thus, Theorem 22(vi) is applicable if, for any $n \in \mathbf{Z}_{+}$,

$$
\begin{aligned}
& K_{T_{n}} \in\left[0, \frac{1}{\sqrt{2}}\right), \quad t_{n} \in[0,1], \\
& K_{T_{n}} \in\left[\frac{1}{\sqrt{2}}, 1\right), \quad t_{n} \in\left[0, t_{n_{1}}\right] \cup\left[t_{n_{2}}, 1\right]
\end{aligned}
$$

or, equivalently, $K_{T_{n}}<1 / \sqrt{1+2 t_{n}\left(1-t_{n}\right)}$ for any given $t_{n} \in$ $[0,1] ; \forall n \in \mathbf{Z}_{0+}$.

Example 24. Assume that $S_{n}=\mu_{n} I, T_{n}=\nu_{n} I$, with $\mu_{n}=\alpha_{n} \nu_{n}$, $K_{S_{n}}=\mu_{n} \in \mathbf{R}_{+}, K_{T_{n}}=\nu_{n} \in \mathbf{R}_{+}$, and $\alpha_{n} \in[0,1] ; \forall n \in \mathbf{Z}_{0+}$ and assume that the metric $d: X \times X \rightarrow \mathbf{R}_{0+}$ is homogeneous and norm-induced, that is, $d(c x, c y)=|c| d(x, y)=|c|\|x-y\|$ for any $c \in \mathbf{R}$ and $x, y \in X$. Assume also that

$$
\begin{aligned}
& \limsup _{n \rightarrow \infty}\left(1-\alpha_{n}-\frac{d\left(x_{n}, x_{n+1}\right)+d\left(x_{n+1}, x_{n+2}\right)}{\left\|x_{n}\right\|+\left\|x_{n+1}\right\|}\right) \\
& \quad \leq 0
\end{aligned}
$$

then

$$
\begin{aligned}
& \limsup _{n \rightarrow \infty}\left(d\left(\alpha_{n} x_{n}, x_{n}\right)+d\left(\alpha_{n} x_{n+1}, x_{n+1}\right)\right. \\
& \left.\quad-d\left(x_{n+2}, x_{n+1}\right)-d\left(x_{n+1}, x_{n}\right)\right) \\
& \quad \leq \limsup _{n \rightarrow \infty}\left(\left(1-\alpha_{n}\right)\left(\left\|x_{n}\right\|+\left\|x_{n+1}\right\|\right)\right. \\
& \left.\quad-d\left(x_{n+2}, x_{n+1}\right)-d\left(x_{n+1}, x_{n}\right)\right) \leq 0 .
\end{aligned}
$$

Note that (60) holds, in particular, if $\left\{\alpha_{n}\right\} \rightarrow 1$. Then, one gets from (57) and (48) that

$$
\begin{aligned}
& d\left(\alpha_{n} v_{n} x_{n+2}, v x_{n}\right)+d\left(\alpha_{n} v_{n} x_{n+1}, v_{n} x_{n}\right) \\
& \quad+d\left(\alpha_{n} v_{n} x_{n+1}, v_{n} x_{n+1}\right)+d\left(\alpha_{n} v_{n} x_{n+2}, v_{n} x_{n+1}\right) \\
& \quad=v_{n}\left(d\left(\alpha_{n} x_{n+2}, x_{n}\right)+d\left(\alpha_{n} x_{n+1}, x_{n}\right)\right. \\
& \left.\quad+d\left(\alpha_{n} x_{n+1}, x_{n+1}\right)+d\left(\alpha_{n} x_{n+2}, x_{n+1}\right)\right) \\
& \quad \leq v_{n}\left(d\left(\alpha_{n} x_{n+2}, \alpha_{n} x_{n+1}\right)+d\left(\alpha_{n} x_{n+1}, x_{n}\right)\right. \\
& \left.+d\left(\alpha_{n} x_{n+1}, x_{n}\right)\right)+v_{n}\left(d\left(\alpha_{n} x_{n+1}, x_{n+1}\right)\right. \\
& \left.\quad+d\left(\alpha_{n} x_{n+1}, x_{n+1}\right)+d\left(\alpha x_{n+2}, \alpha_{n} x_{n+1}\right)\right) \\
& \quad=v_{n}\left(2 \alpha_{n} d\left(x_{n+2}, x_{n+1}\right)+2 d\left(\alpha_{n} x_{n+1}, x_{n}\right)\right. \\
& \left.+2 d\left(\alpha_{n} x_{n+1}, x_{n+1}\right)\right) \leq v_{n}\left(2 \alpha_{n} d\left(x_{n+2}, x_{n+1}\right)\right. \\
& +2 d\left(\alpha_{n} x_{n+1}, \alpha_{n} x_{n}\right)+2 d\left(\alpha_{n} x_{n}, x_{n}\right) \\
& \left.+2 d\left(\alpha_{n} x_{n+1}, x_{n+1}\right)\right)=2 v_{n} \alpha_{n}\left[d\left(x_{n+2}, x_{n+1}\right)\right. \\
& \left.+d\left(x_{n+1}, x_{n}\right)+d\left(\alpha_{n} x_{n}, x_{n}\right)+d\left(\alpha_{n} x_{n+1}, x_{n+1}\right)\right] \\
&
\end{aligned}
$$


and, and from (60) and (61) that

$$
\begin{aligned}
& \limsup _{n \rightarrow \infty}\left(d\left(\alpha_{n} v_{n} x_{n+2}, v x_{n}\right)+d\left(\alpha_{n} v_{n} x_{n+1}, v_{n} x_{n}\right)\right. \\
& \quad+d\left(\alpha_{n} v_{n} x_{n+1}, v_{n} x_{n+1}\right)+d\left(\alpha_{n} v_{n} x_{n+2}, v_{n} x_{n+1}\right) \\
& \left.\quad-8 v_{n} \alpha_{n} \max \left(d\left(x_{n+2}, x_{n+1}\right), d\left(x_{n+1}, x_{n}\right)\right)\right) \leq 0, \\
& \limsup _{n \rightarrow \infty}\left(t_{n}^{2} \alpha_{n}^{2} v_{n}^{2}+\left(1-t_{n}\right)^{2} v_{n}^{2}+8 t_{n}\left(1-t_{n}\right) \alpha_{n}^{2} v_{n}^{2}\right) \\
& \quad<1 \Longrightarrow
\end{aligned}
$$$$
\limsup _{n \rightarrow \infty}\left(\rho_{n}\right)<1
$$$$
r_{1}\left(t_{n}, \alpha_{n}, v_{n}\right)=t_{n}^{2} \alpha_{n}^{2} v_{n}^{2}+v_{n}^{2}+t_{n}^{2} v_{n}^{2}-2 t_{n} v_{n}^{2}+8 t_{n} \alpha_{n}^{2} v_{n}^{2}
$$

$$
\begin{aligned}
& -8 t_{n}^{2} \alpha_{n}^{2} v_{n}^{2}-1=v_{n}^{2}\left(1+t_{n}^{2}-2 t_{n}+8 t_{n} \alpha_{n}^{2}-7 t_{n}^{2} \alpha_{n}^{2}\right) \\
& -1=v_{n}^{2}\left(1+t_{n}^{2}\left(1-7 \alpha_{n}^{2}\right)-2 t_{n}\left(1-4 \alpha_{n}^{2}\right)\right)-1 \\
& <0 ; \quad \forall n \in \mathbf{Z}_{0+}
\end{aligned}
$$

if and only if

$$
\begin{aligned}
r\left(t_{n}, \alpha_{n}, v_{n}\right) & =t_{n}^{2}\left(1-7 \alpha_{n}^{2}\right)+2 t_{n}\left(4 \alpha_{n}^{2}-1\right)+1-v_{n}^{-2} \\
& <0 ; \quad \forall n \in \mathbf{Z}_{0+} .
\end{aligned}
$$

Note that $r\left(t_{n_{1,2}}, \alpha_{n}, v_{n}\right)=0$ if $t_{n_{1,2}}=\left(1-4 \alpha_{n}^{2} \pm\right.$ $\left.\sqrt{\left(1-4 \alpha_{n}^{2}\right)^{2}+\left(1-7 \alpha_{n}^{2}\right)\left(\nu_{n}^{-2}-1\right)}\right) /\left(1-7 \alpha_{n}^{2}\right)$ and (63) holds with $\left\{t_{n}\right\} \subset[0,1]$, and Theorem $22($ vi) is applicable, if for each $n \in \mathbf{Z}_{0+}$,

$$
t_{n} \in \begin{cases}{\left[\max \left(0, \min \left(t_{n_{1}}, t_{n_{2}}\right)\right), \min \left(1, \min \left(t_{n_{1}}, t_{n_{2}}\right)\right)\right]} & \text { if }\left(1-4 \alpha_{n}^{2}\right)^{2}+\left(1-7 \alpha_{n}^{2}\right)\left(v_{n}^{-2}-1\right) \geq 0, \\ {[0,1]} & \text { if }\left(1-4 \alpha_{n}^{2}\right)^{2}+\left(1-7 \alpha_{n}^{2}\right)\left(v_{n}^{-2}-1\right)<0 .\end{cases}
$$

Example 25. A potential application of the given results on motions of robots, or movable bodies in general, on surfaces subject to the definition of admissible obstacle-free corridors is now described. Let $(X, d)$ be a metric space which is a CAT(0) space. Define $z_{t}=t S p \oplus(1-t) T x$ and $\omega_{t}=t S q \oplus$ $(1-t) T y$ for $t \in[0,1]$, which are points of $X$, parameterized by $t$ in $[0,1]$ for any given $x, y, p, q$ being points of $X$, with $S$ and $T$ being nonexpansive mappings on $X$, such that

$$
\begin{aligned}
& d\left(z_{t}, S p\right)=(1-t) d(S p, T x) \\
& d\left(z_{t}, T x\right)=t d(S p, T x) \\
& d\left(\omega_{t}, S q\right)=(1-t) d(S q, T y) \\
& d\left(\omega_{t}, T y\right)=t d(S q, T y) .
\end{aligned}
$$

Define $Q_{t}(x, y)=t x \oplus(1-t) y ; \forall t \in[0,1]$ as in (19a)-(19c) for $x, y \in X$ and $t \in[0,1]$ so that we can characterize the sequences generated via $Q_{t}$ by the Lipschitz-continuous generator mappings $S$ and $T$. The interpretation of these mappings $S$ and $T$ is that they generate sequences of points which define the extreme trajectories for given initial points of obstacle-free corridor to accommodate the trajectories of a movable robot or body, in general. The mappings are defined so that the admissible trajectories are obstacle-free in the admissible motion surface. This obstacle-free motion surface can be modelled, for instance, according to Euclidean buildings, other abstract simplicial complexes or CAT(0) cube complexes. See, for instance, [12-14]. The values of the parameter $t$ in $[0,1]$ allocate the points $z_{t}$ and $\omega_{t}$, and their successive sequential points generated via $Q_{t}$, within segments of $S q$ and $T y$ and their successive points obtained from $S^{n} p$ and $T^{n} x$ for $n \in \mathbf{Z}_{+}$, respectively. Now, Lemma 18 and Theorem 21 can be invoked in this application according to the specific needs. In particular,

(a) if the convergence of the motion trajectory is suited as objective for any given $t$ then the corridor generator mappings $S$ and $T$ on $X$ are defined as appropriate contractive mappings which define the obstacle-free corridor which converges asymptotically to a single trajectory dependent on $t$ taking values in $[0,1]$ [Lemma 18(i)],

(b) if one of the mappings $S$, $T$ is nonexpansive and specifically noncontractive, while the other one is contractive, then the extreme trajectories define a corridor which becomes for a sufficiently large iteration step as narrow as suited accordingly to the initial values of the trajectories definition [Lemma 18(ii)(iii)],

(c) if both mappings are contractive and defined on a nonempty convex closed convex of $X$ then $\left\{Q_{t}\left(S^{m} p\right.\right.$, $T^{n} x$ ) converges (as $m, n \rightarrow \infty$ ) to a unique fixed point for each $t$ which depends on the fixed points of the two generator mappings and the parameter $t$. If one of them is contractive ( $\operatorname{say} T$ ) while the other has a finite number of iterations (say $S$ has $m$ iterations) then $\left\{Q_{t}\left(S^{m} p, T^{n} x\right)\right\}$ converges (as $\left.n \rightarrow \infty\right)$ to a point which depends on $m$, $p$, each value of the parameter $t$ and the fixed point of $T$. Such a $t$-parameterized point becomes to be a function of $m$ as $m$ takes values on $\mathbf{Z}_{+}$ [Theorem 21].

\section{Conflicts of Interest}

The author declares that there are no conflicts of interest. 


\section{Acknowledgments}

The author is very grateful to the Spanish Government and European Fund of Regional Development FEDER for Grant DPI2015-64766-R and to UPV/EHU for Grant PGC 17/33.

\section{References}

[1] B. Piatek, "Halpern iteration in CAT(k) spaces," Acta Mathematica Sinica (English Series), vol. 27, no. 4, pp. 635-646, 2011.

[2] C. Jun, "Ishikawa iteration process," 2013, https://arxiv.org/abs/ 1303.6669.

[3] R. Espínola and A. Fernández-León, "CAT(k) spaces, weak convergence and fixed points," Journal of Mathematical Analysis and Applications, vol. 353, no. 1, pp. 410-427, 2009.

[4] S. Saejung, "Halpern's iteration in CAT(0) spaces," Fixed Point Theory and Applications, vol. 2010, Article ID 471781, 13 pages, 2010.

[5] B. Panyanak and T. Laokul, "On the Ishikawa iteration process in CAT(0) spaces," Bulletin of the Iranian Mathematical Society, vol. 37, no. 4, pp. 185-197, 2011.

[6] A. R. Khan, M. A. Khamsi, and H. Fukhar-ud-din, "Strong convergence in CAT(0) spaces," Nonlinear Analysis. Theory, Methods \& Applications, vol. 74, no. 3, pp. 783-791, 2011.

[7] B. Nanjaras and B. Panyanak, "Demiclosed principle for asymptotically nonexpansive mappings in CAT(0) spaces," Fixed Point Theory and Applications, vol. 2010, Article ID 268780, 14 pages, 2010.

[8] L. Leustean, "A quadratic rate of asymptotic regularity for CAT(0)-spaces," Journal of Mathematical Analysis and Applications, vol. 325, no. 1, pp. 386-399, 2007.

[9] N. Shahzad, "Fixed point results for multimaps in CAT(0) spaces," Topology and Its Applications, vol. 156, no. 5, pp. 9971001, 2009.

[10] G. S. Saluja, "Strong convergence of Noor iteration for generalized asymptotically quasi-nonexpansive mappings in CAT(0) spaces," Acta Mathematica Vietnamica, vol. 37, no. 3, pp. 341355, 2012.

[11] M. A. Hindawi, Asymptotic invariants of hadamard manifolds [Ph.D. dissertation], University of Pennsylvania, 2005.

[12] J. Tits, Buildings of Spherical Type and Finite BN-Pairs, vol. 386 of Lecture Notes in Mathematics, Springer, Berlin, Germany, 1974.

[13] M. Bacák, Introduction to CAT(0) Spaces, CARMA, University of Newcastle, 2010.

[14] M. Bacák, "The proximal point algorithm in metric spaces," Israel Journal of Mathematics, vol. 194, no. 2, pp. 689-701, 2013.

[15] M. Federico Ardila, "La combinatoria de los complejos cúbicos de tipo CAT(0)," in Congreso Colombiano de Matemáticas, July 2011.

[16] M. Kell, "Uniformly convex metric spaces," Analysis and Geometry in Metric Spaces, vol. 2014, no. 2, pp. 359-380, 2014.

[17] W. A. Kirk, "Geodesic geometry and fixed point theory," in Seminar of Mathematical Analysis (MALaga/Seville, 2002/2003), vol. 64 of Colecc. Abierta, pp. 195-225, Universidad de Sevilla, Seville, Spain, 2003.

[18] W. A. Kirk, "Geodesic geometry and fixed point theory. II," in Proceedings of the International Conference on Fixed Point Theory and Applications, pp. 113-142, Yokohama Publisher, 2004.

[19] M. M. Deza and E. Deza, Encyclopedia of Distances, Springer, Berlin, Germany, 2009.
[20] M. Bacák, Convex Analysis and Optimization in Hadamard Spaces, vol. 22 of De Gruyter Series in Nonlinear Analysis and Applications, Walter de Gruyter, Berlin, Germany, 2014.

[21] W. Takahashi, "A convexity in metric space and nonexpansive mappings. I," Kodai Mathematical Seminar Reports, vol. 22, pp. 142-149, 1970.

[22] A. Kaewcharoen and B. Panyanak, "Fixed points for multivalued mappings in uniformly convex metric spaces," International Journal of Mathematics and Mathematical Sciences, vol. 2008, Article ID 163580, 9 pages, 2008.

[23] T. Dominguez Benavides, "Geometric properties of Banach spaces and metric fixed point theory," Extracta Mathematicae, vol. 17, no. 3, pp. 331-349, 2002.

[24] A. Bronsted, "Convex sets and Chebyshev sets," Mathematica Scandinavica, vol. 17, pp. 5-16, 1965.

[25] M. De la Sen and E. Karapinar, "On a cyclic Jungck modified TS-iterative procedure with application examples," Applied Mathematics and Computation, vol. 233, pp. 383-397, 2014.

[26] V. I. Istratesku, Fixed Point Theory. An Introduction, D. Reidel Publishing Company, London, UK, 1983.

[27] V. Berinde, Iterative Approximation of Fixed Points, vol. 1912 of Lecture Notes in Mathematics, Springer, Berlin, Germany, 2007.

[28] M. De la Sen and A. Ibeas, "Properties of convergence of a class of iterative processes generated by sequences of self-mappings with applications to switched dynamic systems," Journal of Inequalities and Applications, vol. 498, 22 pages, 2014. 


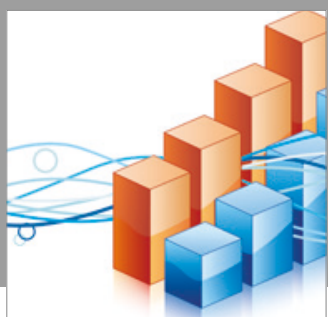

Advances in

Operations Research

vatersals

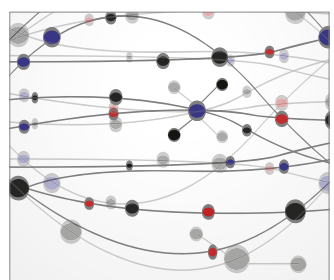

\section{The Scientific} World Journal
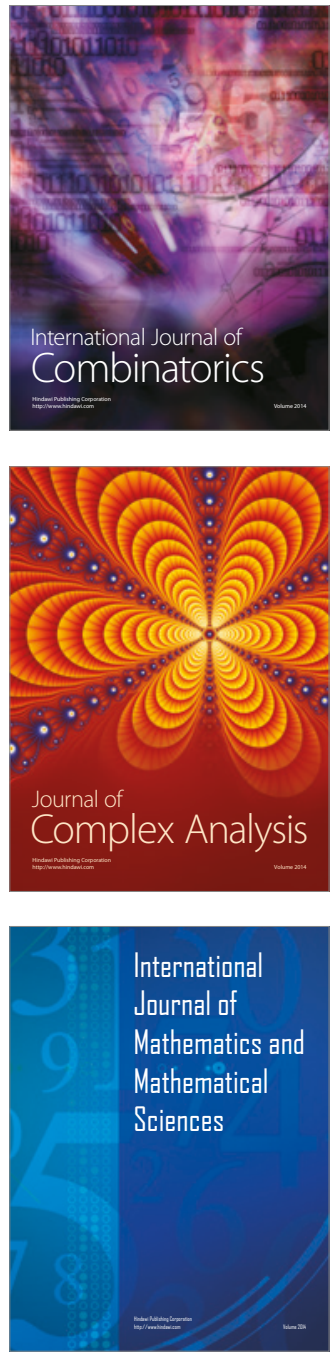
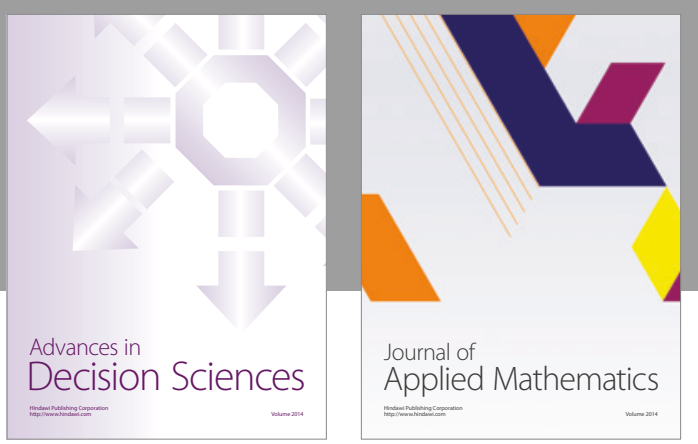

Algebra

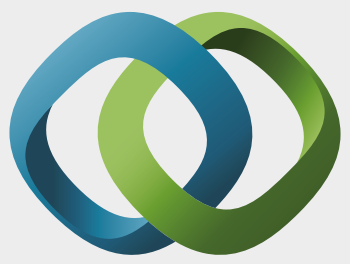

\section{Hindawi}

Submit your manuscripts at

https://www.hindawi.com
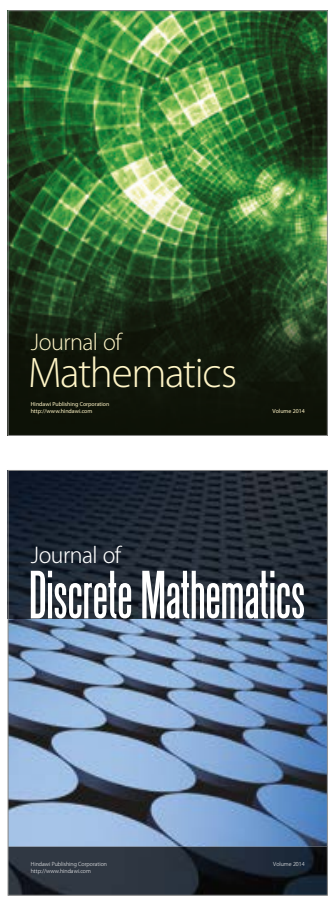

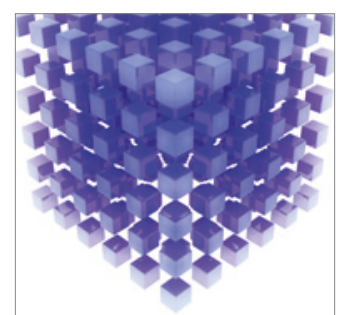

Mathematical Problems in Engineering
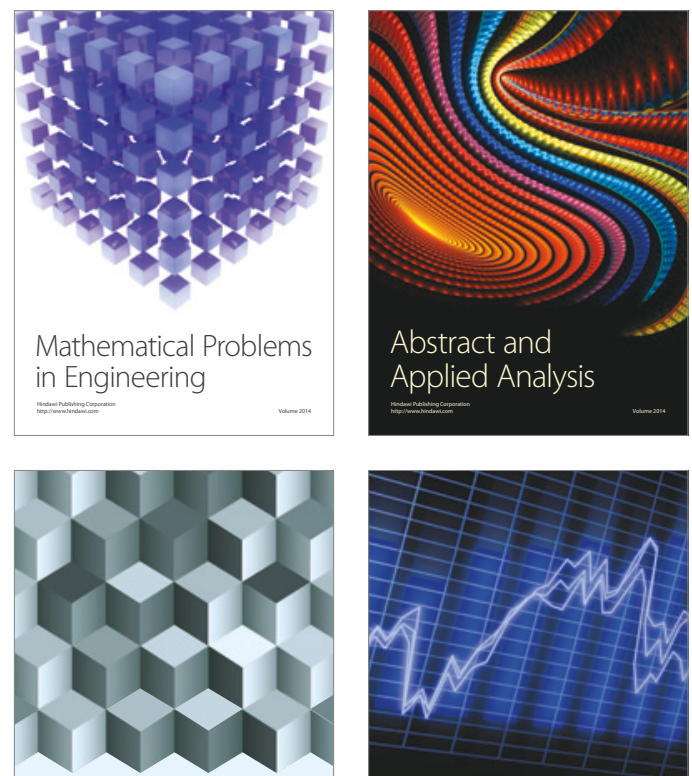

Journal of

Function Spaces

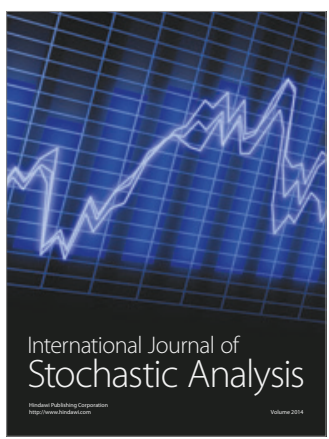

Probability and Statistics
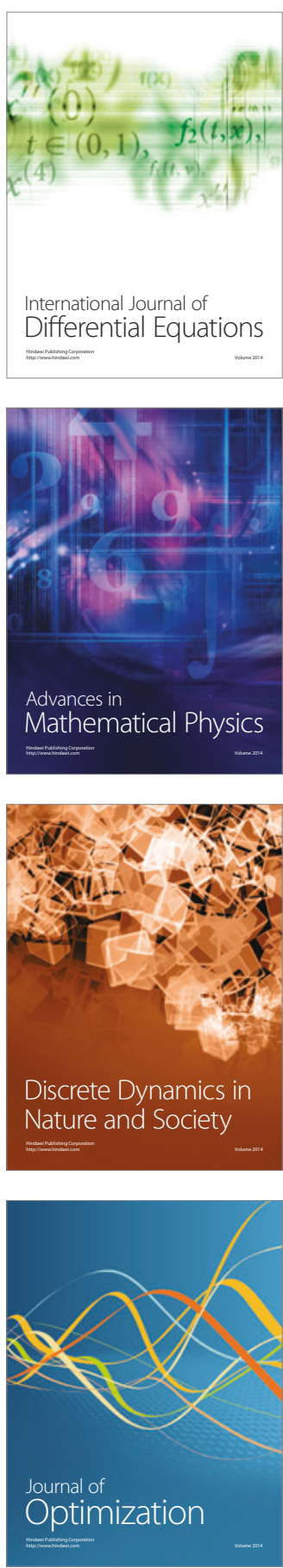